\title{
Convergencia, polarización y movilidad económica. El Salvador, I963-20II
}

Sofía Duarte, Gabriela Guevara, Rodrigo Orantes, Sigfredo Pacheco. Universidad Centroamericana "José Simeón Cañas"

Primer lugar en el concurso de tesis del Departamento de Economía de la UCA, en las Jornadas "Francisco Javier Ibisate", 2014

\section{Resumen}

El presente trabajo busca analizar la desigualdad en El Salvador a través de los conceptos de convergencia, polarización y movilidad, con la intención de motivar el uso de nuevos enfoques para abordar un tema tan complejo. Los resultados, en general, muestran una aparente convergencia regional y disminución de la polarización. Sin embargo, esta información esta sesgada al no contar con datos al interior de los departamentos. Al eliminar a San Salvador, la desigualdad prácticamente permanece en variables como ingreso y productividad. Introduciendo el concepto de polarización se observa que persisten grupos, la movilidad esta en cambio desacelerándose. Por último, las proyecciones de largo plazo mediante matrices de transición muestran que no habrá convergencia regional entre empleo, productividad e ingreso.

\section{Abstract}

This paper analyzes the inequality in El Salvador through notions of convergence, polarization and mobility, with the intention to encourage the use of new approaches to address such a complex issue. The results generally show an apparent decreased regional convergence and polarization. However, this information is biased by not having data within departments. By eliminating San Salvador, inequality remains virtually variables as income and productivity. Introducing the concept of polarization is observed persisting groups, mobility shift was slowing. Finally, longterm projections by transition matrices show that there will be regional convergence: employment, productivity and income.

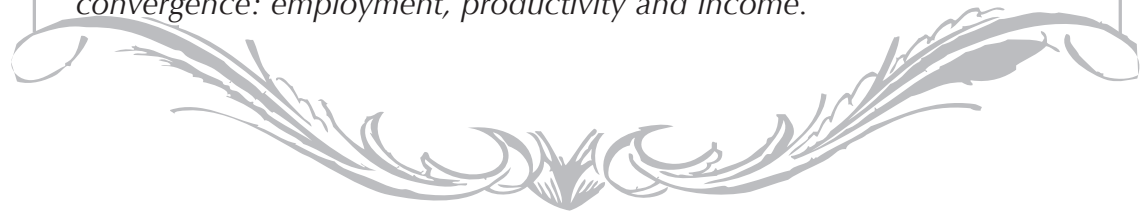




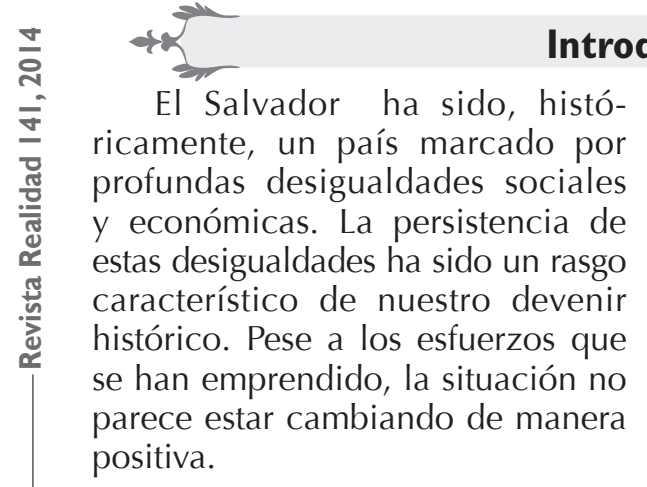

La desigualdad socioeconómica es un problema que acrecienta la brecha entre ricos y pobres en todo estrato social. Nuestra sociedad está marcada por enormes desigualdades, que van desde el ingreso, niveles de empleo, hasta las condiciones de vida de los hogares, etc. Esta situación es alarmante, ya que estas enormes desigualdades entre la población o dentro de los departamentos del país no solo deriva en bajos niveles de calidad de vida de las personas, sino, además, la magnitud de la desigualdad contribuye a reproducir esta situación de una generación a otra. Todas estas modalidades, en que se hace

\section{ducción}

presente la desigualdad (nacional y regional), posibilitan que los Departamentos que actualmente cuentan con dificultades para el desarrollo de las vidas de sus habitantes, continúen careciendo de las condiciones necesarias para mejorar la calidad de vida en el futuro.

En este contexto, surge de manera permanente la necesidad de estudiar la desigualdad económica, actualizando los estudios, extendiendo los periodos de análisis y sobre todo introduciendo conceptos novedosos que vayan más allá de la medición en sí misma de la desigualdad. De ahí la preocupación presente de complementar el estudio habitual de la desigualdad con conceptos de polarización y movilidad, estos dos como características intrincadas del mismo proceso de desigualdad. El primero midiendo la existencia de grupos en una distribución y el segundo midiendo la velocidad con la que cambia la desigualdad.

\section{Contexto histórico de la investigación}

\subsection{Antecedentes}

A partir de los años ochenta surge el interés en el crecimiento económico y la convergencia entre países o regiones. Se define para esta investigación la existencia de convergencia cuando las desigualdades entre países o regiones, en sus niveles de ingreso per cápita, tienden a reducirse en el largo plazo; sin embargo, es necesario distinguir los diferentes tipos de convergencia.
Existe una gran cantidad de investigaciones con respecto a la convergencia en diversos países, especialmente para aquellos desarrollados. El Cuadro 1.1 tiene como propósito resumir algunos de estos estudios, facilitando la búsqueda de trabajos similares sobre los que apoyarse y como un conocimiento general sobre la disponibilidad de información sobre el tema. 


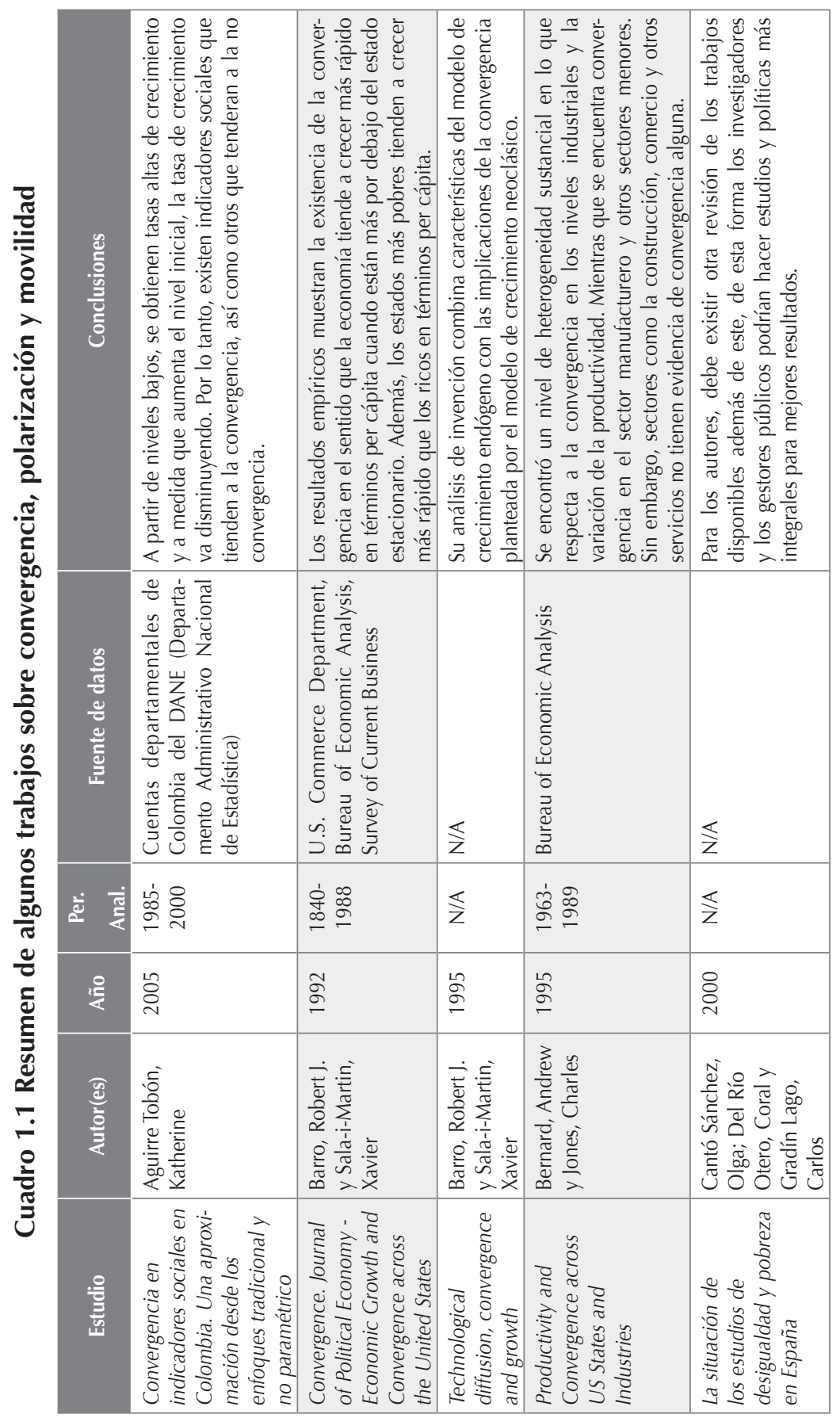




\begin{tabular}{|c|c|c|c|c|c|}
\hline & 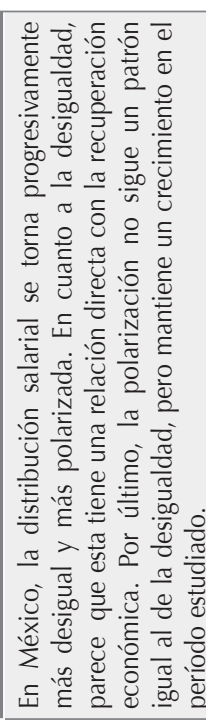 & 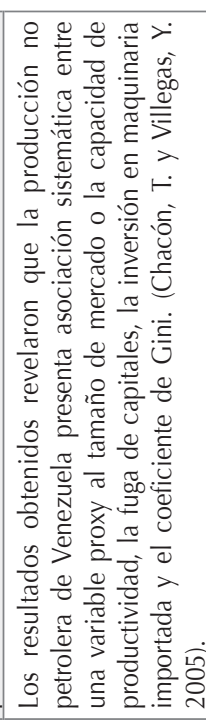 & 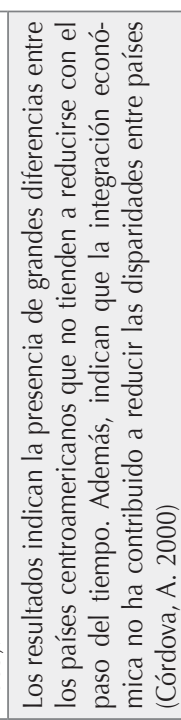 & 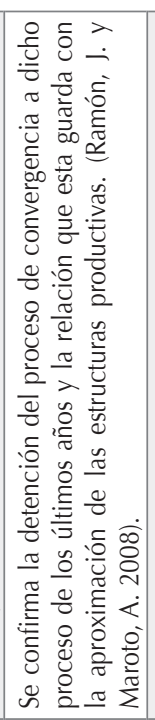 & 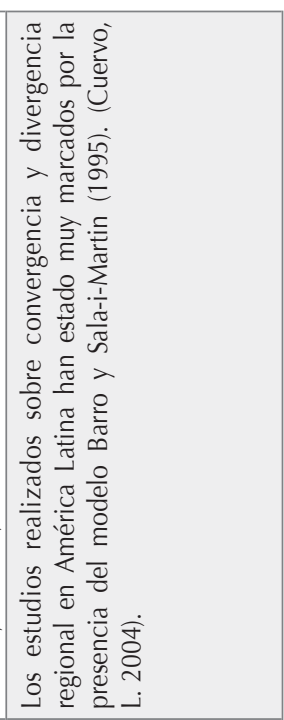 \\
\hline 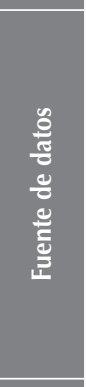 & 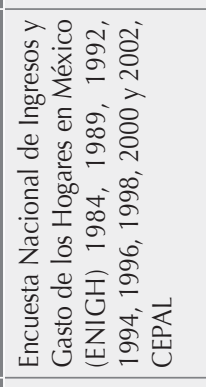 & 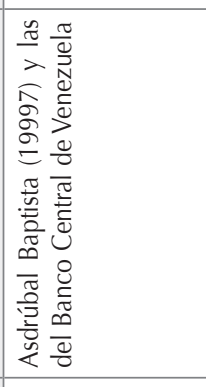 & 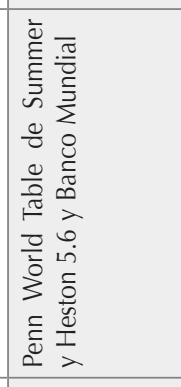 & 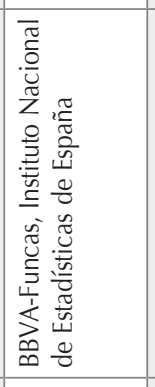 & 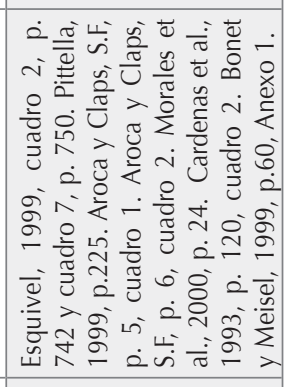 \\
\hline 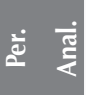 & 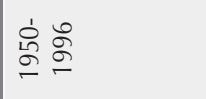 & 宮哭 & 宓哭 & 客 客 & 灾 。ू \\
\hline 営 & 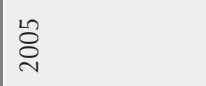 & 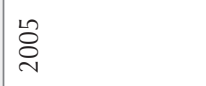 & \&্ণ & 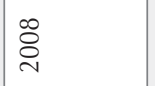 & 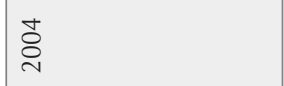 \\
\hline 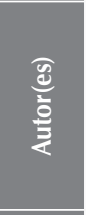 & 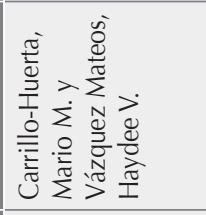 & 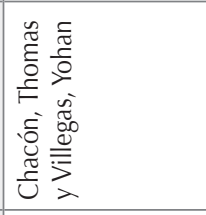 & 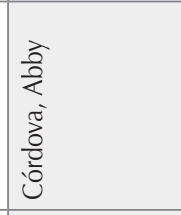 & 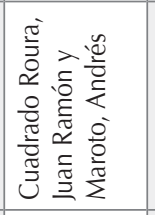 & 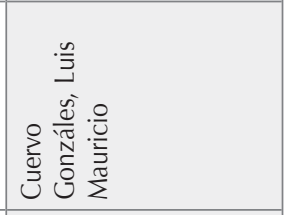 \\
\hline$\frac{\text { 量 }}{\frac{\bar{m}}{4}}$ & 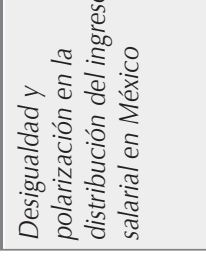 & 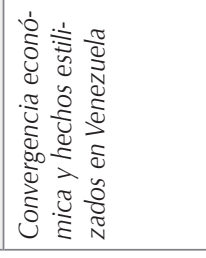 & 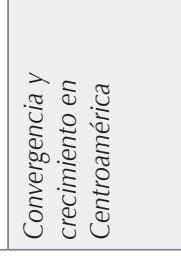 & 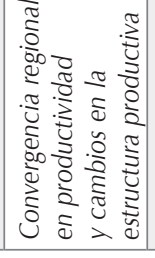 & 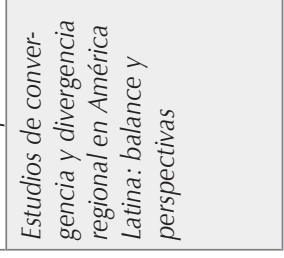 \\
\hline
\end{tabular}




\begin{tabular}{|c|c|c|c|c|c|}
\hline 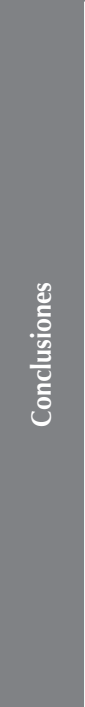 & 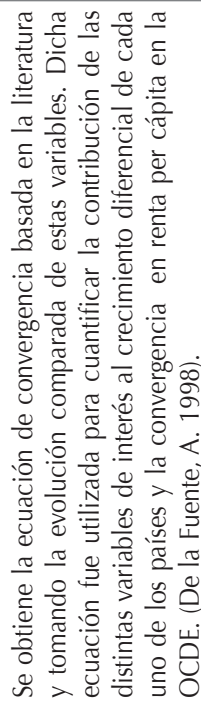 & 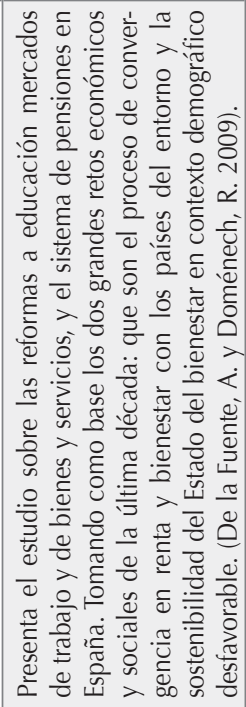 & 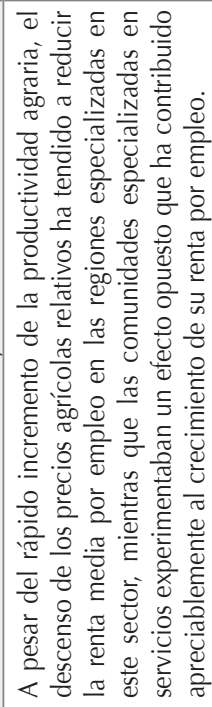 & 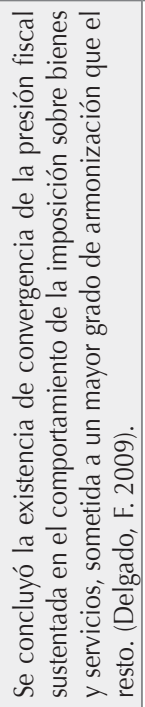 & 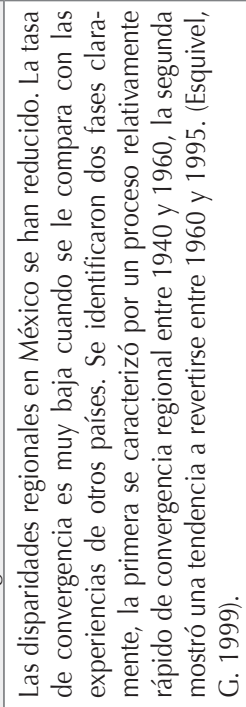 \\
\hline 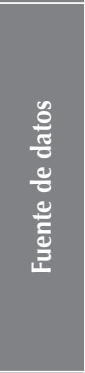 & 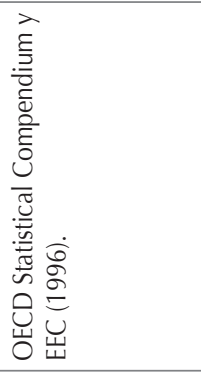 & 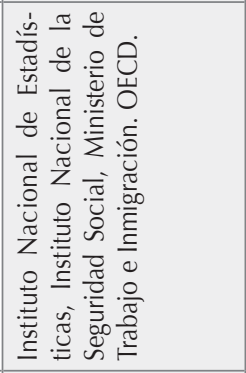 & 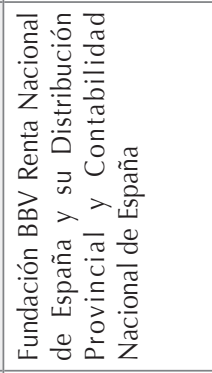 & 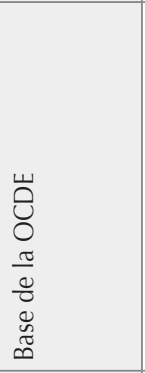 & 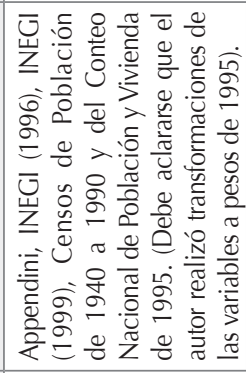 \\
\hline ¿ & 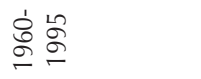 & $\begin{array}{l}1 \\
\infty \\
\circ\end{array}$ & ホ̊ 요 & 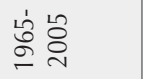 & 守 \\
\hline 题 & $\stackrel{\infty}{\circ}$ & $\stackrel{\text { : }}{\stackrel{1}{2}}$ & 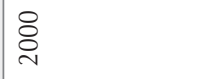 & 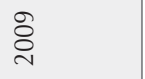 & مू \\
\hline 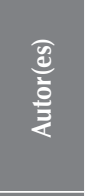 & 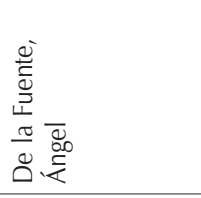 & 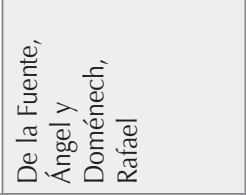 & 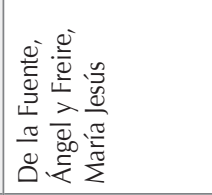 & 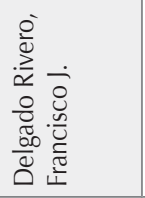 & 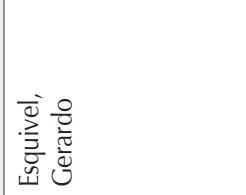 \\
\hline 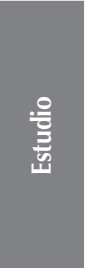 & 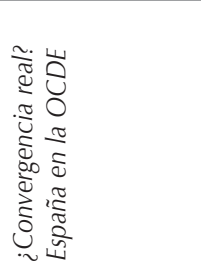 & 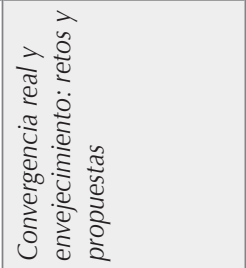 & 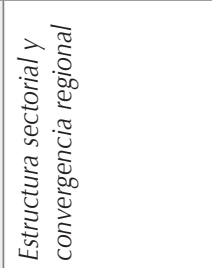 & 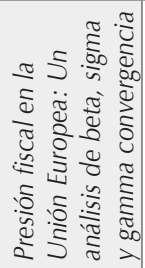 & 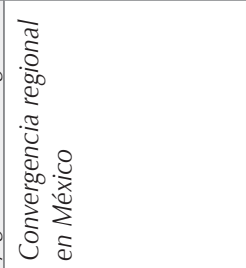 \\
\hline
\end{tabular}




\begin{tabular}{|c|c|c|c|c|c|}
\hline & 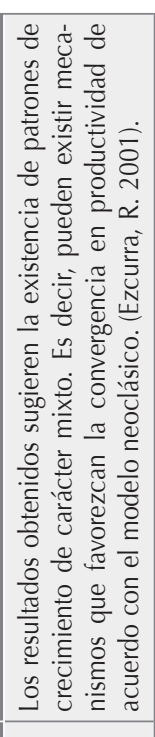 & 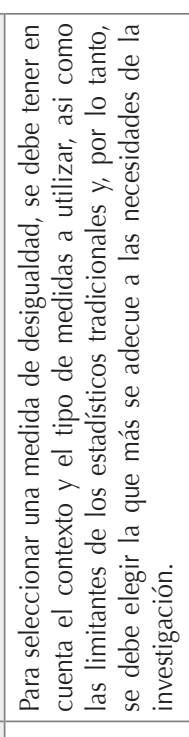 & 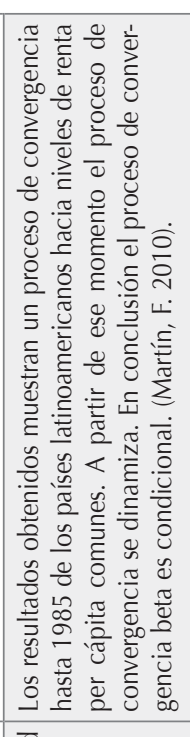 & 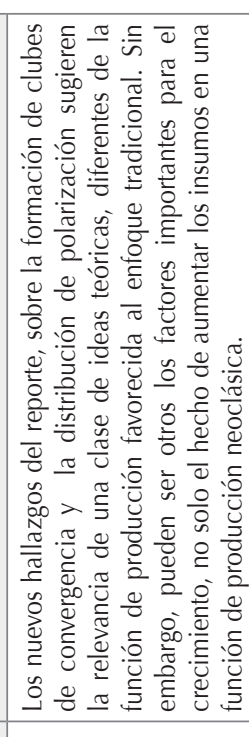 & 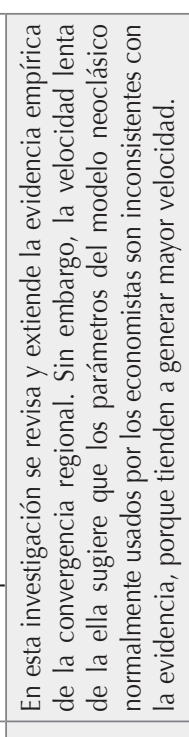 \\
\hline & 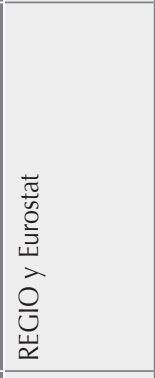 & $\underline{z}$ & 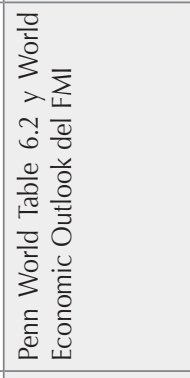 & $\stackrel{\Sigma}{z}$ & $\frac{\pi}{z}$ \\
\hline$\stackrel{d}{\square}$ & $\begin{array}{l}\text { 唐昌 } \\
\text { - }\end{array}$ & $\frac{\tilde{z}}{z}$ & 宫 & $\stackrel{\Sigma}{z}$ & $\stackrel{\Sigma}{z}$ \\
\hline 穻 & $\overline{\mathrm{N}}$ & 足 & $\stackrel{\circ}{\grave{2}}$ & ஓू & 占 \\
\hline 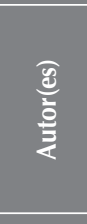 & 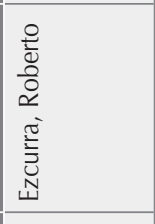 & 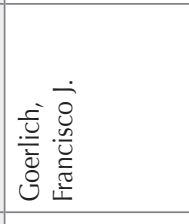 & 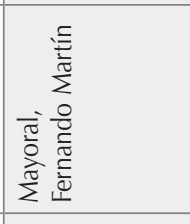 & 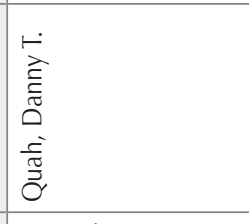 & 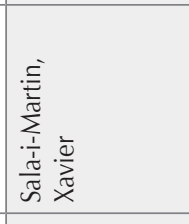 \\
\hline 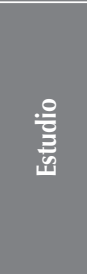 & 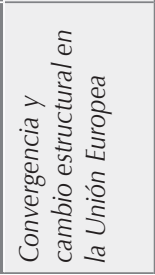 & 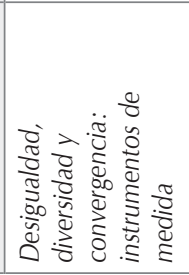 & 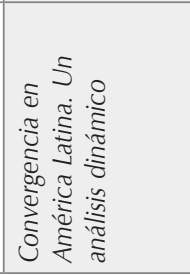 & 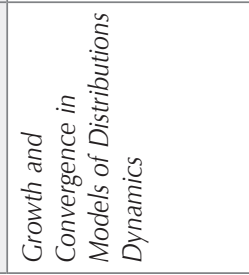 & 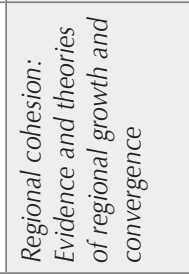 \\
\hline
\end{tabular}




\begin{tabular}{|c|c|c|c|c|c|}
\hline 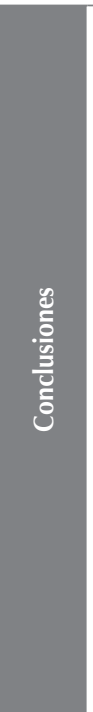 & 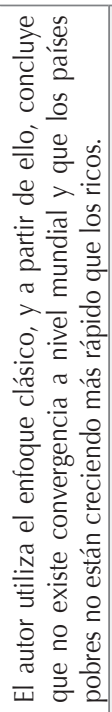 & 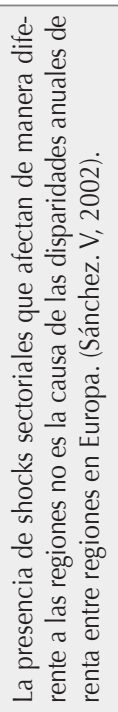 & 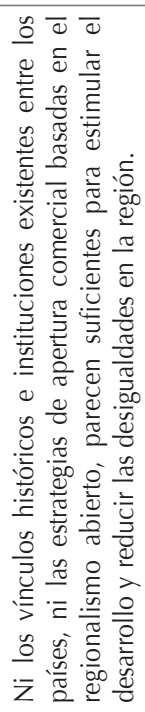 & 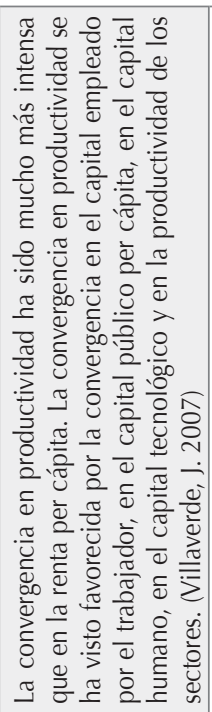 & 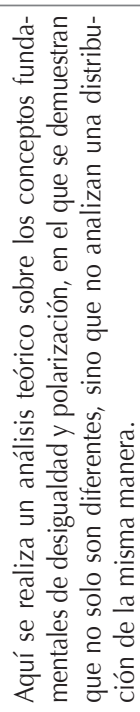 \\
\hline & $\frac{\$}{z}$ & 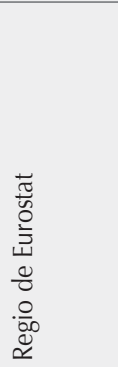 & 窟 & 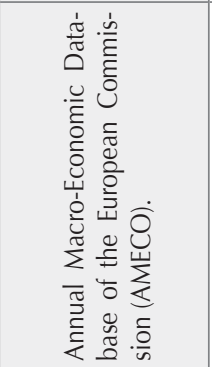 & 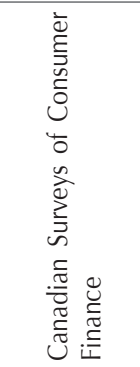 \\
\hline ¿ & $\begin{array}{l}\dot{\delta} \\
\text { ᄋ̊ } \\
\circ\end{array}$ & 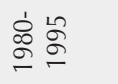 & 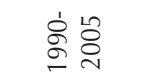 & $\begin{array}{l}\dot{1} \\
\stackrel{8}{\circ} \\
\stackrel{-}{\circ}\end{array}$ & $\begin{array}{l}1 \\
\stackrel{\sigma}{\sigma} \\
\sigma\end{array}$ \\
\hline 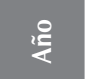 & ஜू & ઠิ & $\frac{0}{2}$ & $\hat{\check{\delta}}$ & ڤे \\
\hline$\frac{\overparen{d}}{\frac{3}{2}}$ & 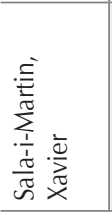 & 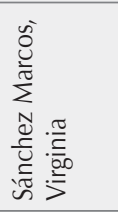 & 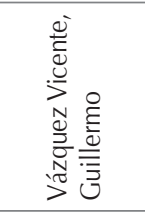 & 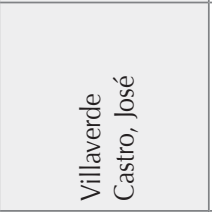 & 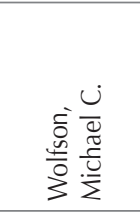 \\
\hline 음 & 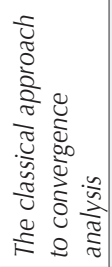 & 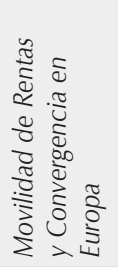 & 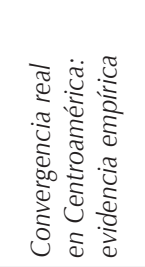 & 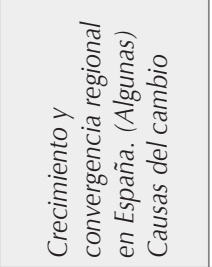 & 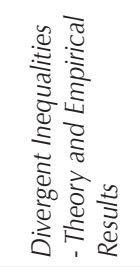 \\
\hline
\end{tabular}


El anterior cuadro resume diversos estudios realizados en el período 1992-2010 que fueron consultados para la presente investigación. Algunos de estos trabajos son solo análisis teóricos de los conceptos, y no presentan un estudio empírico con variables relevantes de algún país. Entre estos, se concluye que se deben estudiar más a fondo los conceptos de convergencia, polarización y movilidad para poder hacer un análisis más completo, y por tanto, con diferentes perspectivas de una misma realidad. En el caso de los estudios que sí realizan un análisis empírico, la mayoría concluye en que existe una convergencia en los países más desarrollados, como Estados Unidos o la Unión Europea. Sin embargo, al compararlos con países como México, El Salvador y la zona centroamericana, se observa que existen grandes diferencias entre estos. Es muy importante destacar que las diferencias entre estos grupos de alto y bajo ingreso no se reducen (como bien lo documenta Quah (1993), y aunque eventualmente existe una convergencia (o mayor igualdad regional) al interior de los países de alto ingreso y entre ellos; este proceso de homologación no se aprecia en los países de bajos ingresos (con mayor población en el mundo). Por lo tanto, para estos últimos países representa un mayor reto converger económicamente y disminuir así sus desigualdades.

Estructura de la investigación. Posterior a la revisión de algunos antecedentes sobre convergencia económica y con el fin de establecer una base teórica que ayude a construir el análisis empírico se comenzará con las características deseables de un índice de concentración, luego se trabajará en los indicadores de polarización y se revisará el concepto de movilidad. Una vez planteados los conceptos y medidas se procederá a realizar en análisis empírico y por último se remarcarán algunas conclusiones.

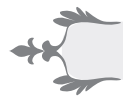

La desigualdad es un concepto de naturaleza eminentemente relativa en tanto que se contrapone al de igualdad, para el cual puede haber más de una definición. Desde el punto de vista estadístico la desigualdad es definida como la concentración de una variable en una población dada de individuos. La desigualdad emerge cuando un individuo o grupo transgrede algún criterio de equidad como es la medida aritmética o se apropia más allá de su participación en el total de individuos o grupos.

Por otra parte, el término desigualdad de la renta hace referencia a la diferencia o disparidad en los niveles de ingreso. Este significado lo adoptó Kuznets (citado en Gradín y Del Río, 2001) en 1953 
al afirmar: "cuando hablamos de desigualdad de la renta, simplemente nos referimos a las diferencias de renta, sin tener en cuenta su deseabilidad como sistema de recompensas o su indeseabilidad como esquema que contradice cierta idea de igualdad" (p. 55).

Sin embargo, el interés en la literatura económica por el concepto de desigualdad no solo se centra en este enfoque, sino que se adentra en los terrenos normativos de la economía. Una segunda acepción la hace el reconocido economista Atkinson quien nos dice que hay "un contenido moral, es decir, existe la presunción de que la igualdad es deseable" (Atkinson, 1987, p. 750), esta línea ética y normativa se extiende en los trabajos de Amartya Sen.

En la actualidad, las economías del mundo han tendido a desarrollarse en el uso de los mercados, bajo la idea de que es a través de ellos que se logra la mejor asignación posible de los recursos productivos $y$, por ende, un mayor crecimiento económico. No obstante, también es conocido, desde las bases de la misma teoría económica, que el mercado no tiene por qué generar una distribución igualitaria o equitativa. A pesar de ello, los críticos de esta corriente de pensamiento continúan argumentando que es posible un crecimiento económico sostenido que genere un mayor nivel de bienestar para el conjunto de la sociedad sin provocar mayores desigualdades en la distribución del ingreso.

En resumen, podemos sintetizar una noción de desigualdad como la distribución de una variable dentro de un grupo específico.

Esta distribución participativa de las unidades tiene aspectos estáticos y dinámicos distinguibles y mensurables (hablamos no solo de un 'estado', sino también de un 'proceso' de concentración): 1. La desigualdad real de la distribución evitando la desigualdad ficticia, provocada por el cambio de escalas con que puede medirse dicha distribución (unidades monetarias, vgr.); 2. La desigualdad absoluta, causada por la apropiación de individuo(s) del grupo, al margen de la equidistribución y 3. La desigualdad relativa, provocada por las transferencias extremas entre individuos, las cuales se distinguen y ponderan más que las transferencias moderadas. Es decir, no es lo mismo que un individuo pobre le ceda ingreso a uno menos pobre que a uno rico, la primera transferencia es moderada y la segunda extrema y debe ser más castigada por el índice de concentración (Sánchez, 2000).

\subsection{Propiedades de los índices}

Comprender el concepto de desigualdad y su medida dependerán de entender la distribución que entraña una cierta variable 
(población, espacio territorial, ingreso, etc.) entre un grupo determinado de individuos, por lo que tener buenos indicadores para medirla dependerá de ciertas características con las que deben contar los índices para tener una apropiada medición y que no generen ambigüedades en el análisis, sino que ayuden a crear fundamentos concretos de las principales razones por las cuales existe desigualdad. Pero, ¿qué deben captar las medidas de concentración? Esto se abordará a continuación.

\subsubsection{Efecto escala}

Evitar el efecto escala en una medida de concentración es algo que implica que esta no deberá sufrir modificación alguna si todas las observaciones han sido afectadas en la misma magnitud. El tamaño de la población no debe ser importante para la medición, sino la proporción en que se dividirá la variable. Esta característica es importante ya que al trabajar la concentración en diferentes grupos, en sus niveles relativos, mantendrá la relación entre estos. Si esto no se toma en cuenta, sucederá que la concentración cambiará en función de las unidades de medida y no por la concentración misma, por tanto la medida (el índice) deberá rechazarse o bien corregirse.

\subsubsection{Pigou-Dalton}

La condición Pigou-Dalton exige que para calificar una medida de desigualdad como un buen indicador, esta debe marcar una caída sistemática siempre que nos aproximemos, a través de redistribuciones sucesivas, a la equidistribución (Cortés y Rubalcava, 1984 p. 28). Por tanto, la condición Pigou-Dalton se refiere a una correspondencia directa entre desigualdad e incremento del índice, es decir que a medida que se incrementa la desigualdad, la medida deberá de incrementarse también (Sánchez, 2000, p. 74).

\subsubsection{Cambio relativo}

Este requisito exige de los indicadores una sensibilidad diferencial para marcar cambios en los grados de concentración según el nivel en que se realicen las transferencias. El índice debe discriminar las formas de transferencias, que van de las unidades más pobres a las más ricas, es decir, las transferencias extremas y las que van de un determinado tipo de unidades a otras inmediatas, transferencia moderada; las unidades deberán estar ordenadas ascendentemente. Así, el índice deberá incrementarse en mayor medida ante transferencias extremas que moderadas. Siendo el coeficiente de Gini un indicador muy utilizado, este aspecto es aún más relevante conceptual y empíricamente en países de América Latina, donde predominan las transferencias extremas ya que precisamente Gini no garantiza esta propiedad (tabla 1.2). Aquí reside la importancia de comprender 
los límites y los retos de medir la desigualdad.

\section{2 Índices de desigualdad (o concentración)}

La medición de la desigualdad a través de índices ha tenido grandes desarrollos metodológicos. Es necesario tener claro el concepto de índice de desigualdad que está definido como una medida que resume la manera de cómo se distribuye una variable entre un conjunto de individuos. Se pueden clasificar dependiendo de su agrupación, las medidas positivas que son las que no hacen referencia a ningún concepto de bienestar social, y las medidas normativas basadas en una función de bienestar. En este apartado se abordan algunas medidas de concentración en base a las propiedades mencionadas anteriormente. En la tabla 1.1., se muestran los índices y su estandarización.

\subsubsection{Rango relativo $\left(R_{R}\right)$}

A partir de la noción de desigualdad podemos suponer que es posible una comparación en magnitud de alguna variable característica entre ciertos individuos, una medición de desigualdad podría ser la diferencia entre la mayor y menor magnitud de las observaciones o individuos, es decir:
Donde $\boldsymbol{\Delta} \boldsymbol{\Delta}$ sugiere un grado de concentración. En consecuencia, en una primera aproximación podemos sostener que a mayor diferencia entre los valores extremos de la variable mayor será el valor de $\boldsymbol{\Delta} \boldsymbol{\Delta}$ y mayor será la desigualdad. A la diferencia entre estas dos observaciones características le denominaremos rango. Cuando exista equidistribución tendremos $X_{\text {máx. }}=X_{\min .} \quad X_{\text {máx. }}=X_{\min .}$ por lo que $R \quad R \quad$ y $R_{N} R_{N}$ serán ambos iguales a cero. Por otra parte, si una unidad posee el total de variable que se reparte entonces $R=R_{\text {max. }}=n \quad R=R_{\text {max. }}=n$, por lo que:

$R_{\text {máx. }}=\frac{X_{m \mathbf{z} x .}-\mathbf{0}}{\bar{X}}=\frac{n \bar{X}}{\bar{X}}=n$

$R_{N}=\frac{R_{m \mathbf{x} x}}{n}=\frac{n}{n}=1$

Este desarrollo de encontrar los valores extremos se realiza en los demás índices (tabla 1.1.). Una desventaja de esta forma de medir la desigualdad es que no recoge toda la información existente en un grupo de individuos. Al aplicar esta medida sobre dos conjuntos de datos es posible obtener un resultado totalmente contradictorio con aquel que se obtiene cuando se toma en cuenta toda la información disponible.

\section{$X_{\text {máxima }}-X_{\text {minima }}=\boldsymbol{\Delta}$}




\subsubsection{Desviación media relativa}

(D)

Esta medida surge del criterio de desigualdad que se basa en comparar el valor de la variable con un valor norma previamente establecida. El valor promedio, puede ser interpretado como una manera de expresar en el lenguaje de la estadística el criterio de "norma democrática".

\subsubsection{Varianza relativa $(\mathrm{V})$}

Una manera de evitar los problemas anteriores (propiedad parcial Pigou-Dalton, motivada por el tipo de desviaciones absolutas), es utilizar la varianza relativa $(V V)$, este índice se calcula tomando cada desviación elevada al cuadrado $\left.\left(d_{1} i^{\mathbf{\top}} \mathbf{2}\right) d_{\mathbf{1}} i^{\mathbf{\top}} \mathbf{2}\right)$. Hasta aquí, esta suma depende de la cantidad de observaciones y al mismo tiempo es sensible a los cambios de escala, se debe corregir por de modo que se llega a establecer como medida de desigualdad.

\subsubsection{Desviación estándar $(\sigma)$}

Al igual que la varianza, la desviación estándar depende del valor medio de la variable. Esto podría ocasionar que una distribución tenga una menor varianza que otra, a pesar de presentar una mayor variación relativa, si es que el ingreso medio de la primera distribución es menor que el de la segunda. Este problema se resuelve utilizando el coeficiente de variación.

\subsubsection{Coeficiente de variación (C.V.)}

El coeficiente de variación es una medida independiente del nivel medio de ingresos y es, además sensible a cualquier transferencia de ingresos en la distribución. Sin embargo, la sensibilidad ante transferencia no depende del valor de $y \cdot y$.

\subsubsection{Varianza de los logaritmos}

$\left(\mathrm{L}^{2}\right)$

Al aplicar transformaciones logarítmicas a los valores de una variable se consigue disminuir la distancia que originalmente existía entre ellos. Esta es una propiedad de los logaritmos, las escalas de los números reales positivos se reducen al incrementarse estos. Estos son proporcionales y conforme crecen linealmente los logaritmos lo hacen de forma decreciente.

\subsubsection{Coeficiente de Gini (G)}

El coeficiente de Gini se definirá como un indicador que se clasifica entre las medidas estadísticas para el análisis de la distribución del ingreso, construido a partir de la curva de Lorenz. Este se diferencia de los presentados con anterioridad en la manera como formaliza la norma democrática. 
En efecto, en lugar de representarla a través del promedio, establece la distribución teórica que debería tener la variable si se repartiese por igual entre todas las unidades.

La idea central que orienta la construcción del índice de Gini consiste en comparar dos distribuciones: la empírica, y la que se deriva de la aplicación de la norma democrática. La comparación se hace operativa a través de las discrepancias entre las frecuencias relativas acumuladas de la variable en ambas distribuciones (ver tabla 1.1).

\subsection{8 Índice $\left(R_{M} R_{M}\right)$}

Cuando la transferencia se hace desde una unidad rica a una pobre, el coeficiente de desigualdad $R_{M} R_{M}$ experimenta una caída mayor que si se hace en favor de una observación no tan pobre. Por tanto, podemos afirmar que este coeficiente de desigualdad cumple con la condición Pigou-Dalton y a la vez es sensible al nivel de la variable en que se realiza la redistribución ${ }^{1}$. Este es un buen índice de concentración, ya que reúne todas las condiciones ideales de las medidas de concentración, puede estandarizarse sin problema alguno. En la siguiente parte del trabajo se expondrá un índice que, cumpliendo todas estas características, tiene una mayor sensibilidad que $R_{M} R_{M}$, Theil. Ambos, además, pueden descomponerse por lo que para el análisis regional tienen una clara ventaja.

\subsection{9 Índice entrópico de Theil $(\mathrm{H})$}

Esta medida de concentración no cambia su valor si todos los valores de la variable son afectados constantemente. A la vez, cumple con las condiciones de PigouDalton y con las de sensibilidad a las transferencias de valores de variable ubicados en distintos niveles (cambio relativo). El hecho de que la entropía responda a los dos últimos criterios antes mencionados nos garantiza que entre los valores máximo y mínimo la función sea creciente al aumentar los niveles de desigualdad. 


\begin{tabular}{|c|c|c|}
\hline Índices de desigualdad & Limites (Mx́ximo y mínimo) & Estandarización \\
\hline$R=X_{\text {matema }}-X_{\text {resuma }}$ & $R_{M=}=\sum x_{i}=\bar{x} n ; R_{m i 0}=0$ & $R_{s}=R / \sum x_{i} ; 0 \leq R_{i} \leq 1$ \\
\hline$R_{R}=\frac{X_{\text {matims }}-X_{\text {minnox }}}{\bar{x}}=\frac{D}{\bar{x}}$ & $R_{R_{\text {IIII }}}=n, R_{R_{\text {mox }}}=0$ & $R_{\mathrm{Rt}}=\frac{R_{\mathrm{R}}}{n} \quad 0 \leq R_{\mathrm{Rr}} \leq 1$ \\
\hline$D=\sum_{j=1}^{n} \frac{\left|x_{1}-\bar{x}\right|}{n \bar{x}}$ & $D_{u}=\frac{2(n-1)}{n} D_{-}-0$ & $\begin{array}{c}D_{c}=\frac{D}{D_{u \Delta}}=\frac{1}{2(n-1)} \frac{\sum_{i=1}^{\mid}\left|x_{i}-\bar{x}\right|}{\bar{x}} \\
0 \leq D_{c} \leq 1\end{array}$ \\
\hline$\nabla=\frac{\sum_{i=1}^{\infty}\left(x_{1}-\bar{x}\right)^{2} / m}{\bar{x}^{-1}}$ & $V_{d(\mathrm{II}}=(n-1), V_{\min }=0$ & $\begin{array}{c}V_{c}=\frac{\sigma^{2}}{(n-1)^{-2}} \\
0 \leq V_{s} \leq 1\end{array}$ \\
\hline$L^{ \pm}=\sum_{\mathrm{j}=1}^{\mathrm{n}}\left(\ln x_{1}-\ln \bar{x}\right)^{2} / n$ & $\begin{array}{c}L_{M \text { III }}^{2} \text { es indeteminado: } \\
I_{\text {mio }}^{2}=0\end{array}$ & No se puede estandarizar \\
\hline$G=\sum_{i=1}^{n-1}\left(P_{i}-Q_{i}\right) / \sum_{i=1}^{n-1} P_{i}$ & $G_{M x}=1 ; G_{\min }=0$ & $0 \leq \leqslant \leq 1$ \\
\hline$\left(\frac{q_{i}}{p_{i}}\right)$ & 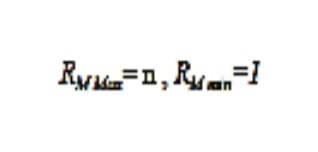 & $\begin{array}{c}R_{M H_{c}}=\frac{R_{m}-1}{n-1} \\
0 \leq R m_{\mathrm{r}} \leq 1\end{array}$ \\
\hline$C V .=\frac{\sigma}{\bar{X}}=\frac{\sqrt{\sum_{1=S}\left(x_{i}-\bar{x}\right)^{2} / n}}{\bar{X}}$ & $\begin{array}{l}C . V_{\operatorname{sen}}=\sqrt{1-n} \\
C . V_{\min }=0\end{array}$ & $\begin{array}{c}C . V_{\mathrm{s}}=\frac{C V .}{\sqrt{1-n}} \\
0 \leq C . V_{.} \leq 1\end{array}$ \\
\hline$\sigma^{2}=\sum_{j=1}^{n}\left(x_{i}-\bar{x}\right)^{2} / n$ & $\sigma^{2} \omega_{\pi}=(n-1) \bar{x}^{2} ; \sigma_{\min }^{2}=0$ & $\begin{array}{c}\sigma^{2}=\sigma^{2} /(n-1) \bar{x}^{2} \\
0 \leq \sigma^{2} \leq 1\end{array}$ \\
\hline$N=\sum\left|y_{i}-l_{i}\right| ; Z=\sum\left|y_{i}-I_{i}\right|\left(y_{i} / l_{t}\right)$ & 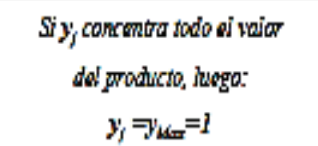 & $\begin{array}{l}0 \leq N \leq 2\left(1-l_{\text {Mar }}\right) \\
0 \leq Z \leq\left(1 / l_{\text {Mer }}\right)-1\end{array}$ \\
\hline$H^{\prime}=\dot{\sum}_{i=1} q_{j} \ln \left(\frac{q_{i}}{p_{1}}\right)$ & $\mathrm{H}_{\mathrm{ML2}}^{\prime}=\infty, \mathrm{H}_{\min }^{\prime}=0$ & No se puede estandarizar \\
\hline
\end{tabular}

Fuente: Elaboración propia con base en artículos varios. 
A continuación se presenta una tabla resumen de las medidas de desigualdad estudiadas, en cuanto al cumplimiento de las condiciones ideales de las medidas de concentración.

\section{Tabla 1.2 Resumen del cumplimiento de las condiciones ideales de las medidas de concentración (datos no agrupados)}

\begin{tabular}{|c|c|c|c|c|}
\hline \multicolumn{5}{|c|}{ Bondades de los Índices de desigualdad } \\
\hline & & Cumple & Cumple & \\
\hline Índice & $\begin{array}{c}\text { Supera } \\
\text { Efecto Escala }\end{array}$ & $\begin{array}{c}\text { Condición } \\
\text { Pigou-Dalton }\end{array}$ & $\begin{array}{c}\text { Condición } \\
\text { Cambio Relativo }\end{array}$ & $\begin{array}{c}\text { Se puede } \\
\text { estandarizar }\end{array}$ \\
\hline Rango relativo & Sí & No & No & Sí \\
\hline Desv. media relativa & Sí & Solo parcialmente & No & Sí \\
\hline Varianza relativa & Sí & Sí & No & Sí \\
\hline Varianza de logaritmos & Sí & Sí & Sí & No \\
\hline Gini & Sí & Sí & No siempre & Sí \\
\hline Índice RM & Sí & Sí & Sí & Sí \\
\hline Theil & Sí & Sí & Sí & Sí \\
\hline
\end{tabular}

Fuente: Elaboración propia, a partir de varios artículos.

\subsubsection{0. Índice de Atkinson}

Los juicios de valor sobre la desigualdad siempre tienen algún contenido normativo, ya sea explícito o implícito. Esta es la razón por la que algunos autores prefieren partir de la interpretación de la desigualdad como una pérdida potencial en el bienestar colectivo y a partir de una función de bienestar social, que refleje de forma explícita los juicios de valor acerca de la relación entre desigualdad y bienestar. Si bien fue Dalton (1920) el primer autor que argumentó que cualquier medida de desigualdad debe estar referida al bienestar económico, es la familia de índices normativos de Atkinson la más comúnmente utilizada (Goerlich, 1998).

Dado un volumen de renta total $Y Y$, Atkinson define la renta igualitaria equivalente, $\mu_{e} \mu_{e}$, como aquel nivel de renta per cápita tal que, si es disfrutado por toda la población, generaría el mismo nivel de bienestar social que la distribución inicial de renta. 


$$
A(\varepsilon) \begin{cases}1-\left[\sum p_{i}\left(\frac{x_{i}}{\mu}\right)^{1-\varepsilon}\right]^{\frac{1}{1-\varepsilon}} & \varepsilon \neq 1 \\ 1-\exp \left[\sum p_{i} \log \left(\frac{x_{i}}{\mu}\right)\right]=1-\Pi\left(\frac{x_{i}}{\mu}\right)^{p_{i}} & \varepsilon=\mathbf{1}\end{cases}
$$

Obsérvese además que $A$ (1) $=1-(\tilde{\mu} / \mu)$. Cuando $\varepsilon=0$, $\varepsilon=0$, es decir no hay aversión social a la desigualdad, $A(\mathbf{0})=0$, $A(0)=0$, por lo que el valor social de la desigualdad es nula cualquiera que sea la distribución de la renta. Para $\varepsilon>0 \quad \varepsilon>0$ y distribuciones continuas el índice $A(\varepsilon) A(\varepsilon)$ varía entre 0 , igualdad perfecta y 1 , máxima desigualdad.

\section{Polarización}

El estudio del concepto y medición de polarización es esencialmente diferente al de desigualdad. Supóngase una población de individuos agrupada en conjuntos de acuerdo a un vector de características, de manera que cada uno de estos es similar en términos de los atributos de sus miembros, pero existen miembros con atributos muy diferentes dentro del conjunto. En ese caso, se dice que la sociedad está polarizada (Esteban y Ray, 1994).

Básicamente, un estudio de polarización busca "estudiar en qué medida se están conformando en la distribución diferentes polos" (Gradín, 2000, p. 423). Entonces, la idea detrás del concepto de polarización es que esta se da cuando existe una distribución fraccionada en grupos que son internamente homogéneos. Y se da una máxima polarización cuando solo existen dos grupos, cada uno con la mitad de la población y se encuentran en los extremos de la distribución.

Es evidente, que para autores como Joan-Maria Esteban, Debraj Ray, Jean-Yves Duclos (2004), Michael Wolfson, entre otros, existe un gran número de fenómenos que pueden explicarse de mejor manera al tener el enfoque de un análisis de polarización y no solo el de desigualdad. Por esto su insistencia en el desarrollo de una medida de polarización y lo que esta puede llegar a implicar en una sociedad determinada. Los siguientes diagramas hacen comprender el concepto de polarización.

1. Debe existir un alto grado de homogeneidad dentro de cada grupo (intragrupos) 
Diagrama 1.1.

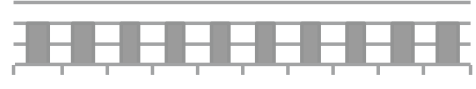

$\begin{array}{llllllllll}1 & 2 & 3 & 4 & 5 & 6 & 7 & 8 & 9 & 10\end{array}$

Esquema a

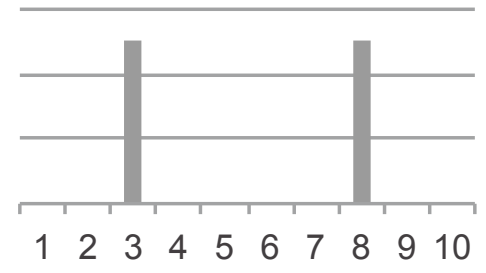

Esquema b

2. Debe existir un alto grado de heterogeneidad a través de cada grupo (intergrupos)

\section{Diagrama 1.2.}

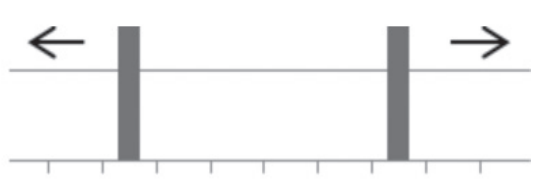

$\begin{array}{llllllllll}1 & 2 & 3 & 4 & 5 & 6 & 7 & 8 & 9 & 10\end{array}$

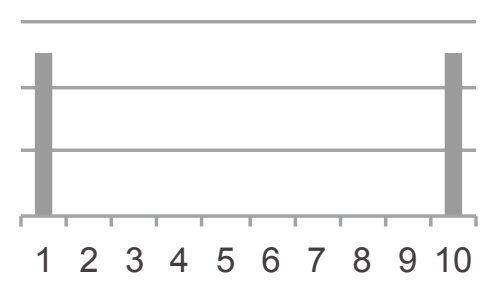

Esquema b

3. Debe haber un pequeño número de grupos con un tamaño significativo. En particular, los grupos con un tamaño insignificante tienen poco peso (por ejemplo, individuos aislados).

\section{Diagrama 1.3.}

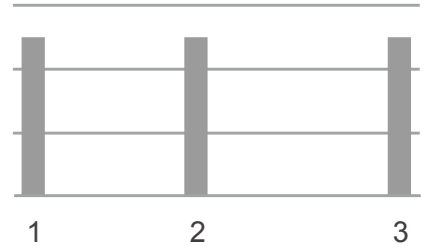

Esquema a

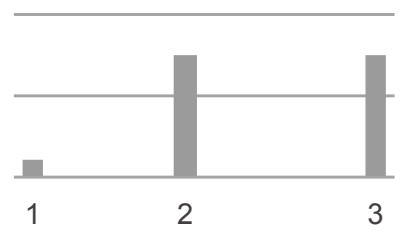

Esquema b

Fuente: elaboración propia con base en Esteban y Ray (1994) p. 826 
Con los esquemas anteriores se ilustra la última característica. En el esquema a, la población está distribuida en tres puntos y sus atributos tienen la misma distancia. Considerando a los grupos 2 y 3 aproximadamente del mismo tamaño, al darse un movimiento de un pequeño grupo de la población, del grupo 1 al 3, se podría notar rápidamente un problema en comparación con los ejemplos anteriores. Dicho movimiento tiene como resultado la definición muy clara de dos grupos y la posibilidad del surgimiento de tensiones sociales por parte del grupo restante.

\subsection{Esteban y Ray}

Una medida de polarización $P^{*}$, es de la forma:

$$
(E R)=P^{*}(\pi, y)=K \sum_{i=1}^{n} \sum_{j=1}^{n} \pi_{i}^{1+\alpha} \pi_{j}\left|y_{i}-y_{j}\right|
$$

Para algunas contantes $K>\mathbf{0} K>\mathbf{0}$ y $\alpha \in\left(0, \alpha^{*}\right) \alpha \in\left(0, \alpha^{*}\right)$, donde

$$
\alpha^{*} \cong 1.6 \alpha^{*} \cong 1.6 \text {. }
$$

\subsection{Wolfson}

Wolfson construye una curva de polarización en un análisis paralelo a la curva de Lorenz y el coeficiente de Gini. Empezando por la noción de una dualidad entre desigualdad y polarización, existe un desarrollo para ambos casos a partir de una función acumulativa de densidad para una distribución de ingresos.

\section{Gráfico 2.8 Nueva medida de polarización de Wolfson basado en la Curva de Lorenz}

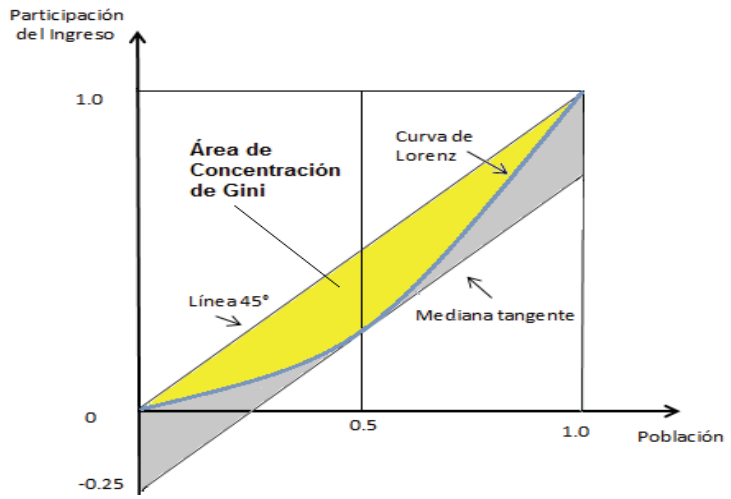

Fuente: Elaboración propia con base en Wolfson, M. (1994) p.355 
Matemáticamente esto se puede expresar de la siguiente manera: siendo $F$ una distribución de ingreso de $n n$ individuos, con una media del ingreso equivalente a $\mu \mu$ y la mediana $m$

$$
P_{m}^{W}(F)=\mathbf{4} \frac{\mu}{m} P_{1}=4 \frac{\mu}{m}\left[T_{m}-\frac{G(F)}{2}\right]=2 \frac{\mu}{m}[2(0.5-L(0.05))-G(F)]
$$

Es importante notar que, a pesar de tener una relación con el índice de Gini y la curva de Lorenz, Wolfson está de acuerdo con la diferencia que existe entre el concepto de polarización y desigualdad. Wolfson concluye expresando que entre los años 1973-1981 las medidas consistentes con la curva de Lorenz disminuyen o se mantienen constantes, mientras que la polarización aumentó. Y además, que al existir una divergencia entre ambos conceptos, es necesario incluir en una investigación de la distribución del ingreso medidas relacionadas con polarización.

\section{3. Índice de Zhang y Kanbur}

De la misma manera en que Esteban, Ray y Wolfson proponen una medida de polarización, Zhang y Kanbur desarrollaron un estudio para el caso de China en el que introducen una nueva y mejor (según su criterio) medida de polarización. Están de acuerdo con que existen diferencias entre el concepto de desigualdad y el de polarización. Sin embargo, se preguntan si las medidas de polarización ya propuestas dan una mejor respuesta a este problema a lo largo del tiempo.
Si las diferencias en el nivel de ingreso dentro de un grupo (intragrupal) disminuyen este se vuelve más homogéneo. En términos relativos, esto quiere decir que las diferencias entre los grupos van a verse incrementadas y por tanto la polarización también. En el caso que las medias de ambos grupos sean más desiguales, la polarización aumenta. De esta forma Zhang y Kanbur escriben el índice de polarización de la siguiente manera:

$$
P=\frac{\text { desigualdad intergrupal }}{\text { desigualdad intragrupal }}
$$

Este índice propuesto por Zhang y Kanbur parece ofrecer una forma de análisis de la desigualdad descompuesto y basta con tomar la desigualdad con Theil o Rm tal como se ha planteado en los índices de concentración. Obteniendo la desigualdad entre grupos e intragrupos. Esto permite ver a mayor profundidad los cambios en la distribución del ingreso desde dos perspectivas diferentes que a la vez están relacionadas. En el caso analizado por los autores, se encontró que China en términos de polarización es mayor en el ámbito urbano-rural, sin embargo 
el incremento es más drástico en el caso del interior y la costa. Así concluyen que este análisis refleja las verdaderas preocupaciones políticas actuales con su nueva medida de polarización.

Hemos presentado dos características que nos ayudan a estudiar la desigualdad en una sociedad en términos económicos, las cuales son concentración y polarización. Pero necesitamos analizar la movilidad que existe dentro de los polos (expuestos en polarización). Debemos determinar si existe movilidad en la distribución y en qué medida.

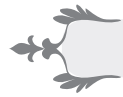

La movilidad explica la dinámica de los ingresos, puede existir movilidad creciente y decreciente de hogares o de regiones. Se puede tener un nivel de desigualdad en el periodo " $\mathrm{t}$ " y disminuir ésta para el periodo " $\mathrm{t}+\mathrm{s}$ ", pero otra distribución puede llegar a ese mismo nivel de desigualdad en la mitad de tiempo. Por otro lado, el caso de El Salvador es interesante, se tiene que regionalmente Gini es alrededor del $15 \%$ pero en los últimos años la movilidad ha disminuido (Gráfica 1.1 y 1.4). La consecuencia de este análisis es útil y obvia: será más difícil que aumente la igualdad regional en los próximos años.

La movilidad se puede distinguir por sus diferentes fenómenos temporales, los cuales son:

- Movilidad a lo largo del ciclo de vida.

- Movilidad en un corto plazo ligado a la coyuntura económica.

- Movilidad entre generaciones.
Existen diferentes tipos de indicadores sintéticos que nos ayudan a calcular la movilidad entre ellos tenemos.

El coeficiente de correlación que nos presenta el grado de sensibilidad de los valores extremos entre grupos, lo cual provoca que este sea poco usado. La solución para un análisis de sensibilidad es el indicador del coeficiente Spearman, ya que este realiza el análisis por medio de rangos.

Otro indicador que podemos encontrar es la matriz de transición que es aquella que relaciona la distribución de los hogares según clase, y esta puede ser en cuartiles, deciles, quintiles, etc., cuya relación se da de un año $t$ a un año $t+k$. Cabe decir que la matriz de transición es estocástica debido a que sus filas suman uno.

El índice de inmovilidad es aquella proporción de los hogares que no presentan cambios en sus niveles de ingresos, es decir, la 
proporción promedio de hogares que se sitúan en la diagonal principal de la matriz de transición.

Definir el estado de inmovilidad y de movilidad perfecta nos ayuda a ver el grado de movilidad que proviene del análisis de las matrices de movilidad. Habrá inmovilidad perfecta cuando en ninguno de los hogares se vea un cambio de la clase en el periodo $\mathrm{t}+\mathrm{k}$ respecto a la clase que corresponde al periodo t. La inmovilidad total se verá sobre la diagonal principal de la matriz y esta concentre todos los hogares. La movilidad perfecta se dará cuando el hogar tenga una igual probabilidad de localizarse en un quintil, sin importar el quintil inicial. Por medio de la matriz transición podemos encontrar la distribución ergódica que es aquella que nos ayuda en el largo plazo a observar si una distribución se convierte en estacionaria, sabiendo que su distribución inicial no lo era. El vector ergódico nos ayudará a observar la proporción de los hogares que se sitúan por debajo, muy cercanos y por encima de la media de los hogares, es decir, que podemos ver en el largo plazo si existirá movilidad o no.

Otro aspecto importante dentro de la movilidad es conocer el grado de movilidad y para ello podemos ayudarnos de los siguientes índices.

El test de Pearson chi-cuadrado es aquel que compara la matriz de transición esperada bajo la hipótesis de independencia con la matriz de transición observada. En las cuales se ven los elementos de la matriz, $\mathrm{Eij}=\left(\mathrm{Ei}^{*} \mathrm{Ej}\right) / \mathrm{n}$, donde $\mathrm{n}$ es el número de observación y $\mathrm{Ei}$ y Ej son las frecuencias marginales de las columnas y filas de la matriz observada. Específicamente para matriz de transición en quintiles.

Luego tenemos el índice Cramer, que nos ayuda a ver la asociación de los hogares por medio de una escala que permite analizar el grado de movilidad. Si el índice toma un valor de cero tendremos movilidad perfecta y si este toma un valor de 1 , tendremos inmovilidad perfecta.

Existen dos índices que son los más utilizados para analizar la movilidad que ellos involucran por aparte de los porcentajes de la diagonal principal como los porcentajes que se sitúan alrededor de la diagonal principal, lo cual nos ayuda a hacer un análisis más profundo de nuestra matriz de transición.

El primero de ellos es el índice de Shorrocks, este nos ayuda a saber la probabilidad de permanecer en el mismo estado. Si obtenemos un índice cercano a cero podemos decir que la probabilidad de que el individuo se sitúe en el mismo estado crece y si el índice se acerca a uno podemos decir que la probabilidad de que el individuo transite entre los estados es mayor. 
El segundo índice es un complemento del índice de Shorrocks: es el índice de Bartholomew, el cual promedia los movimientos de las transiciones que se dan alrededor de la diagonal principal, es decir, que nos ayudaría a ver las transiciones a estados de mejor condición o peor condición del individuo comparándolo con su estado inicial.
En El Salvador, al igual que en otros países de América Latina, las diferencias de ingreso y de oportunidades entre ricos y pobres son mayores que las diferencias en sus niveles de satisfacción de la vida. Por ejemplo, según el Informe del PNUD para El Salvador, 2012, la concentración de ingreso por hogares fue del $43 \%$ en ese año y en 2007 fue del 48\%, existe pues una mayor igualdad. Sin embargo, dada la teorización anterior mostrando la insuficiencia de la concentración, la hipótesis de este trabajo es que esta información no es concluyente y esto se aplicará a la dimensión regional y particularmente departamental. Se demostrará que hay matices que establecer y enfatizar al introducir el concepto de movilidad y polarización. Este es ahora el reto del análisis empírico.

Es por esto que se desarrolla un análisis sobre la situación de la desigualdad regional en El Salvador en el período de 1998 a 2011. Primero, las medidas de concentración anteriormente planteadas ayudan a ver la evolución de la desigualdad. Segundo, se mide el aspecto de la polarización con los índices indicados, que, como sabemos, ofrece una mejor explicación sobre lo que está sucediendo dentro de los grupos y entre ellos mismos en una sociedad. Posteriormente se analiza la movilidad de estas distribuciones.

En las sociedades modernas, el crecimiento económico ha servido como medida del éxito o del fracaso de las políticas gubernamentales y consecuentemente, de la búsqueda del bienestar y la felicidad de las personas. Así, el producto interno bruto (PIB) es el indicador clave de los avances o retrocesos de un país. No obstante, debe tenerse claro que un aumento en los ingresos no incrementa automática o proporcionalmente el bienestar de las personas. Este no es un límite estadístico, sino una de las variables utilizadas. El trabajo actual no resolverá esta discusión y asumirá que el producto per cápita reflejará aunque sea imperfectamente el nivel de vida regional, ya que al menos existe la noción de que hay una fuerte correlación entre estos dos conceptos. Una variable más sensible es la productividad ya 
que muestra el concepto de producción material más fielmente.

El ingreso per cápita muestra que en el país, a nivel departamental y en términos de concentración, se tiende a la convergencia, es decir que la desigualdad en esta variable disminuye. Ya que al observar el comportamiento del índice de Theil ha disminuido en casi un 3\% en el período 19982011, esto lo muestra la gráfica 1.1.
Esta aseveración no se ve afectada por el índice utilizado. $Y$ debe aclararse que los diferentes índices muestran una clara caída. También debe advertirse que no tiene sentido comparar los niveles de los índices, pues como se ha conceptualizado con anterioridad, su construcción implica diferentes características. En cualquier caso, la conclusión es clara, independientemente de las características de la concentración, ésta regionalmente ha disminuido.

\section{Gráfico 1.1. Concentración departamental de Ingreso Per cápita El Salvador 1998-2011, mensual. Índices varios.}

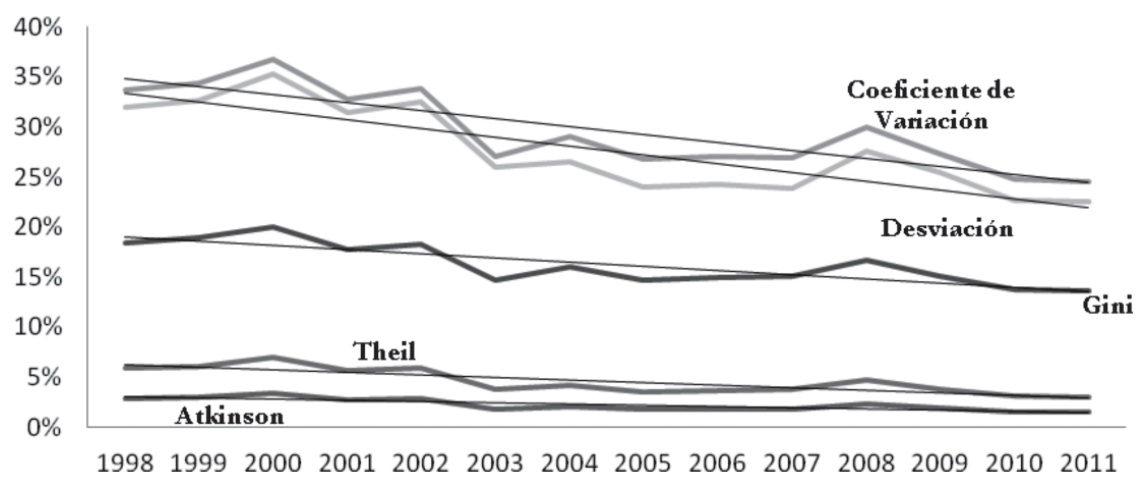

Fuente: Elaboración propia a partir de datos de la Encuesta de Hogares de Propósitos Múltiples, EHPM, (DIGESTYC 1998-2011).

Por consiguiente, la disminución de la desigualdad implica, en estricto sentido que, a nivel departamental existe una ligera mejoría en el ingreso por persona. Pero solo eso. Si los datos por departamento se descompusieran por municipios esto mostraría con mayor fidelidad la igualdad entre personas y departamentos, pues se descompondría la desigualdad entre departamentos y al interior de ellos. La experiencia en estos cálculos muestra que el mayor nivel de desigualdad se encuentra al interior de la unidad regional, es decir, del departamento y no entre los departamentos. Desafortunadamente, esta información municipal no está disponible para todos los años. Pero precisamente por ello es importante aclarar los límites y alcances del anterior resultado. 
Siguiendo con la idea de recuperar la mayor información posible de los datos departamentales y no municipales. En términos de polarización el índice que se utilizó es el índice de Zhang y Kanbur. De igual forma que el índice de Theil, este índice es el que cumple con las mejores características para realizar un análisis de polarización. En el período de análisis el índice presenta una tendencia hacia la baja, disminuyendo en un $20 \%$, lo que nos llevaría a decir que a nivel departamental los grupos que se presentan en la distribución están en un proceso de homologación, y más estricta y técnicamente hablando, de "desagrupación", ver gráfico 1.2.

Nuevamente, es importante notar que se realizó un análisis, tanto en términos de concentración y polarización, calculando los índices de Theil y Zhang y Kanbur excluyendo solo al departamento de San Salvador. Esto es justificado ya que es el departamento que presenta la mayor cantidad de población y también los mayores niveles de ingreso per cápita del país. Es pues interesante controlar y confrontar esta dicotomía.

\section{Gráfico 1.2. Polarización Departamental de Ingreso Per cápita El Salvador 1998-2011, mensual. Índices varios}

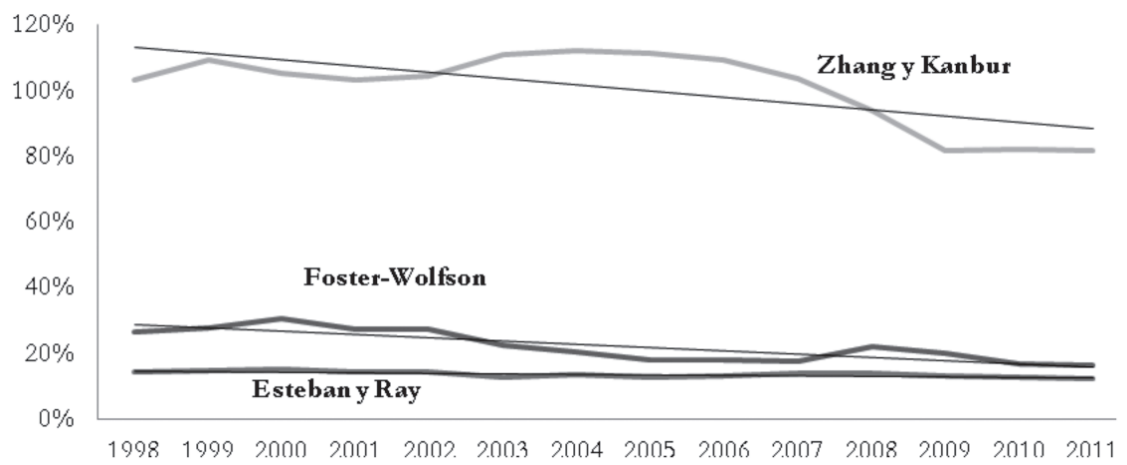

Fuente: Elaboración propia a partir de datos de EHPM (DIGESTYC, 1998-2011).

En términos de concentración pudimos observar que San Salvador provoca que exista un mayor nivel de desigualdad; ya que en el año 1998 se observó que el índice de Theil sin San Salvador es de un 4\%, aproximadamente, en comparación al $6 \%$ que obtuvimos analizando todos los departamentos. En el año 2011 se observa el mismo comportamiento, ya que el índice de Theil sin San Salvador es de un 2\% en comparación al índice con todos los departamentos que es de un $3 \%$. 
Pero aquí puede rescatarse un matiz muy interesante. Si los 13 departamentos restantes fueran un solo país, la mayor igualdad aparente no es tan pronunciada. Esto puede observarse claramente porque la caída del indicador es menor en la gráfica 1.3 sin San Salvador. En otras palabras, ese país virtual sin San Salvador no va hacia la igualdad con la misma velocidad que con este departamento. Además se aprecia que existió una mayor desigualdad regional de 2005-2008.

Los "dos países" (con y sin San Salvador) nos permiten ver lo que sucede más al interior de los departamentos. El análisis de polarización, en un estudio más amplio, nos muestra que realizando el análisis sin San Salvador existe un mayor grado de polarización. Ya que se observa una tendencia creciente en comparación a la tendencia decreciente que se observó en análisis con todos los departamentos. Es decir, que podríamos pensar que en el resto de los departamentos la polarización se encuentra más marcada.

Se realizó otro tipo de análisis de polarización, calculando el índice de Zhang y Kanbur, comparando San Salvador contra el resto de departamentos, según los resultados concurre una polarización campo/ciudad muy marcada. Estas "dos realidades" de El Salvador, ciudad-campo, no cambian según lo muestra el gráfico de agrupaciones (gráfico 1.4 b), la realidad persistente es "San Salvador y los demás departamentos".

\section{Gráfico 1.3. Concentración Departamental del Ingreso Per cápita. El Salvador. 1998-2011, mensual. Índice de Theil.}

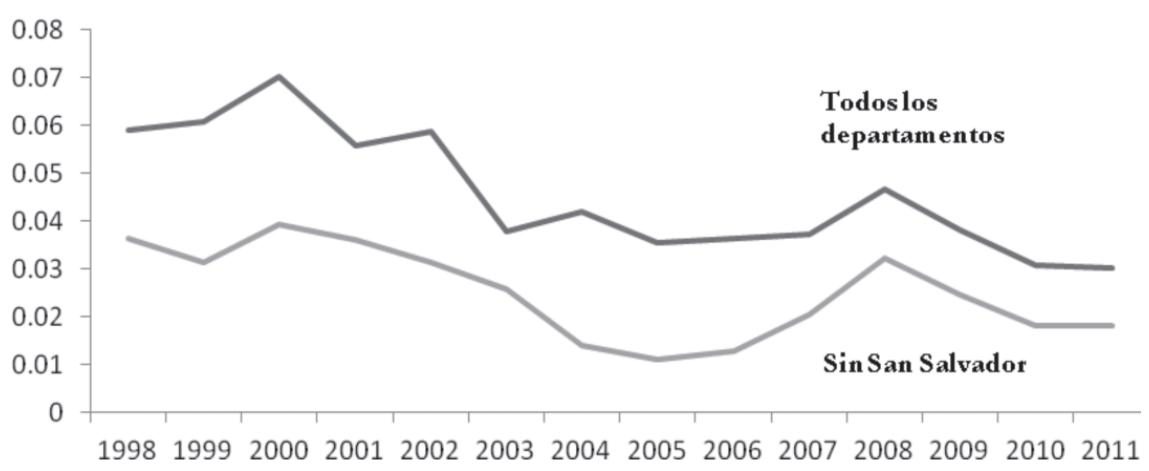

Fuente: Elaboración propia a partir de datos de EHPM (DIGESTYC, 1998-2011). 
Para complementar nuestro análisis, ya que hemos observado que la desigualdad en términos de concentración ha disminuido y hemos probado la presencia de grupos en dicha distribución, es necesario conocer, ahora, la movilidad de la distribución. La movilidad, tomando como medida la media salvadoreña, podemos concluir que en el período de 19982011 ha existido y esta ha tendido a aumentar durante dicho período; esto según los índices de Shorrocks y Bartholomew (gráfico 1.4 a). Pero debe atenderse que esta movilidad está desacelerando. Por otro lado, la matriz de transición en su diagonal muestra la movilidad (si sus valores tienden a 1 hay menos movilidad) y este instrumento nos ayuda a consolidar la afirmación que en el largo plazo el $45 \%(0.13+0.15+0.17)$ de los departamentos no tenderá a la media nacional, 22\% estarán en ella y $33 \%$ por encima.

\section{Matriz de transición 1. Ingreso Per cápita. El Salvador, 1998-2011 Intervalos, \% respecto a la media de El Salvador}

\begin{tabular}{|c|c|c|c|c|c|}
\hline & $0-65 \%$ & $65 \%-73 \%$ & $73 \%-79 \%$ & $79 \%-92 \%$ & $92 \%-\infty$ \\
\hline 38 & 0.61 & 0.29 & 0.08 & 0.03 & 0.00 \\
\hline 38 & 0.26 & 0.37 & 0.32 & 0.03 & 0.03 \\
\hline 37 & 0.08 & 0.22 & 0.35 & 0.32 & 0.03 \\
\hline 33 & 0.00 & 0.03 & 0.21 & 0.64 & 0.12 \\
\hline 36 & 0.00 & 0.03 & 0.03 & 0.06 & 0.89 \\
\hline \multicolumn{6}{|c|}{182 transiciones (13 años $x 14$ depart.) } \\
\hline Distribución Ergódica & 0.13 & 0.15 & 0.17 & 0.22 & 0.33 \\
\hline
\end{tabular}

Fuente: Elaboración propia a partir de datos de EHPM (DIGESTYC, 1998-2011).

Nota: La lectura es la siguiente: de 38 ocasiones en que un departamento estaba en el intervalo de $0 \%-65 \%$ de la renta media salvadoreña, $61 \%$ de esas 38 situaciones se mantuvieron en ese intervalo, 29\% escalaron al intervalo de $65 \%-73 \%$, $8 \%$ al de $73 \%-79 \%$, etc. Como consecuencia, la traza media de la matriz o la suma promedio de las celdas diagonales nos expresa la movilidad regional del ingreso per cápita. La matriz sirve también para calcular la distribución de largo plazo y mediante, la traza y sus complementos, los indicadores de movilidad de Shorrocks y Bartholomew. 
Movilidad y agrupaciones departamentales de Ingreso Per cápita. El Salvador 1998-2011.

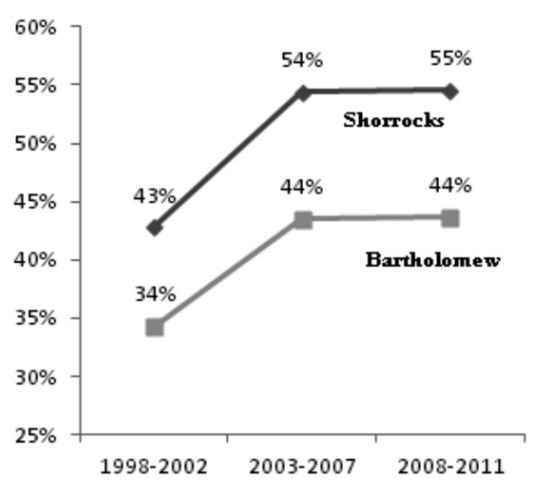

Desaceleración de movilidad, mismas agrupaciones, mayor ingreso

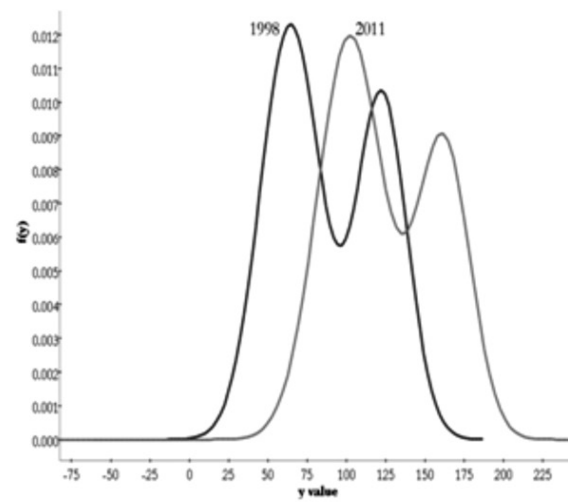

Fuente: Elaboración propia a partir de datos de EHPM (DIGESTYC, 1998-2011). Se trata del ingreso per cápita mensual.

La siguiente variable que estudiaremos es la productividad, ya hemos indicado que esta variable manifiesta la producción material más fielmente que el PIB per cápita. En términos de concentración la variable presenta una tendencia a la convergencia, si centramos la lectura en el índice de Theil dadas sus características teorizadas. La desigualdad en términos de productividad está disminuyendo en El Salvador, ya que el índice de 1998 a 2011 ha decrecido aproximadamente en $15 \%$ (gráfica 1.5.). Departamentalmente, entonces, se produce cada vez más homogéneamente. Debe advertirse, en cambio, que los niveles de desigualdad regional son más altos en productividad que en ingreso, aunque ciertamente la velocidad de decrecimiento aquí fue mayor. Pero esta lectura también es importante enfatizarla, ya que, la productividad es la base sobre la que posteriormente se podrá distribuir el ingreso por persona. 
Gráfico 1.5

Concentración departamental de productividad El Salvador 1998-2011. Índices varios

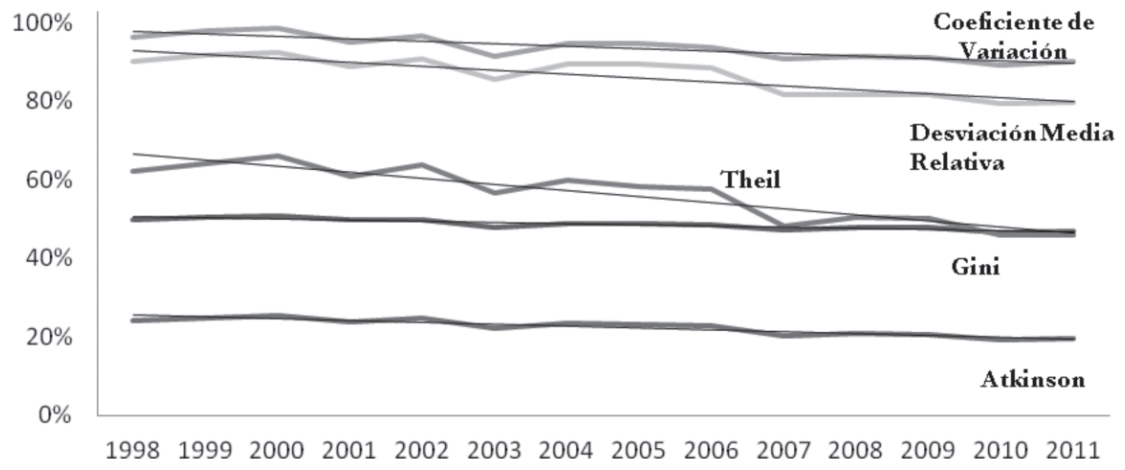

Fuente: Elaboración propia a partir de datos de EHPM (DIGESTYC, 1998-2011).

\section{Gráfico 1.6.}

Polarización departamental de productividad. El Salvador 1998-2011. Índice Zhang y Kanbur

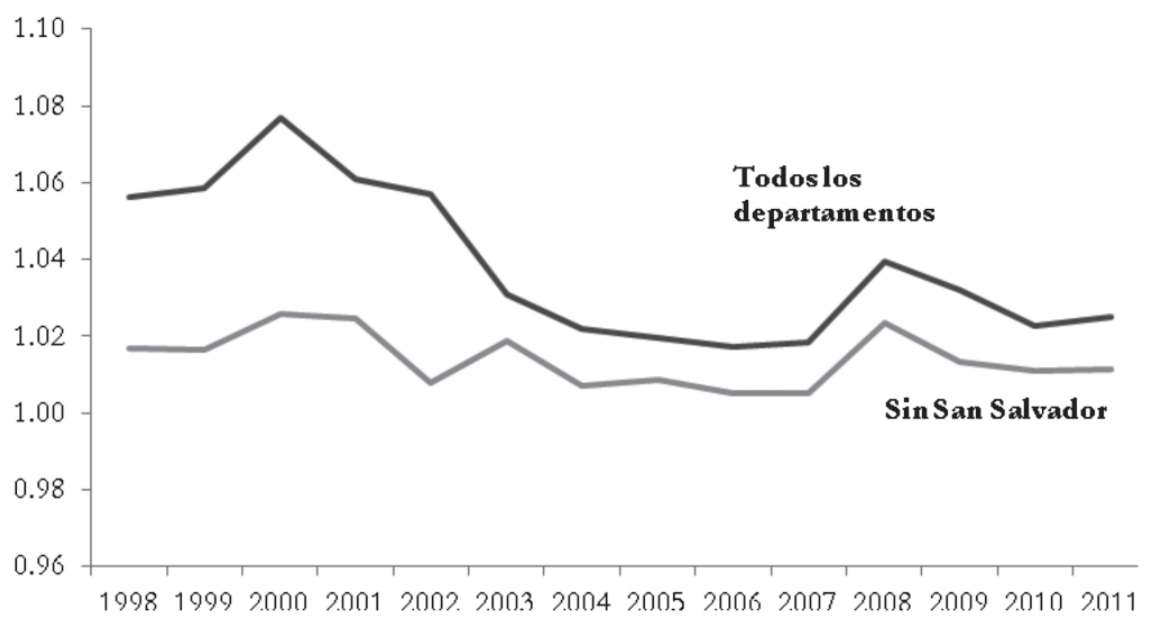

Fuente: Elaboración propia a partir de datos de EHPM (DIGESTYC, 1998-2011). 
Gráfico 1.7 a y b

Movilidad y grupos departamentales de productividad. El Salvador

1998-2011.

Menor movilidad estructural, mismas agrupaciones y niveles de productividad.
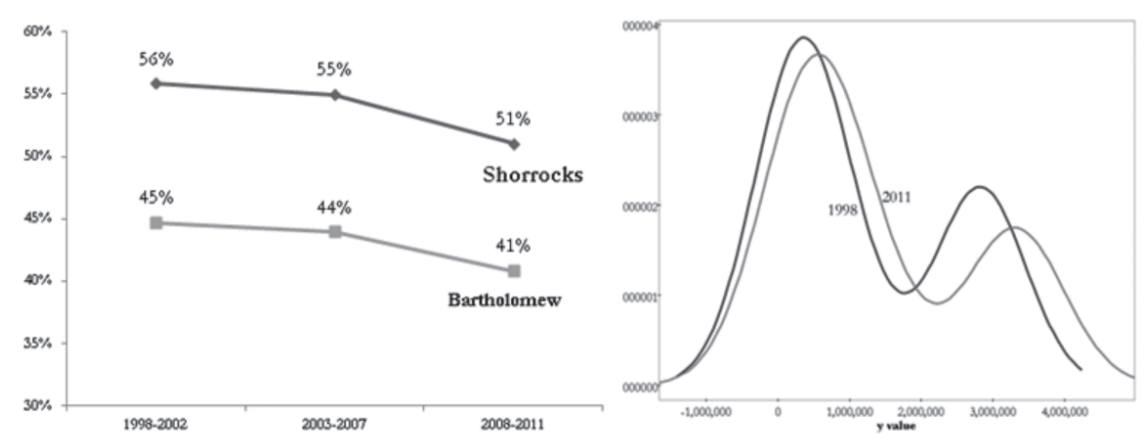

Fuente: Elaboración propia a partir de datos de EHPM (DIGESTYC, 1998-2011).

Hay que aclarar que cuando se asegura que la desigualdad está disminuyendo no significa que la productividad del país esté creciendo o mejorando, si no que se estaría presentando un proceso de homogenización.

El análisis de polarización nos muestra que la formación de grupos dentro de la distribución existe y que dicha polarización ha disminuido por lo que los grupos se están homogenizando. Ya que en el período de 1998-2011 el índice de Zhang y Kanbur ha disminuido en 3\% aproximadamente. Al igual que en el análisis de ingreso per cápita se realizó un análisis tanto en términos de concentración y polarización calculando los índices de Theil y Zhang y Kanbur excluyendo de él, el departamento de San Salvador (gráfica 1.6.).
De ese modo, se muestra que eliminándolo, prácticamente no hay cambio, siguen existiendo los mismos grupos.

En términos de concentración (en el estudio más amplio), pudimos observar que San Salvador provoca que exista un mayor grado de desigualdad. Ya que en el año 1998 se ha observado que el índice de Theil sin San Salvador es de un 22\% aproximadamente en comparación al $62 \%$ que obtuvimos analizando todos los departamentos. En el año 2011 se observa el mismo comportamiento ya que el índice de Theil sin San Salvador es de un $16 \%$ en comparación al índice con todos los departamentos que es de un $46 \%$.

El análisis de polarización, como se comentaba, nos muestra que realizando el análisis sin San 
Salvador provoca un mayor grado de polarización, ya que se observa una tendencia creciente en comparación a la tendencia decreciente que se observó en análisis con todos los departamentos. Es decir, que podríamos pensar que en el resto de los departamentos la polarización está más marcada.

De la misma manera que en el ingreso per cápita en la variable productividad se realizó el análisis de polarización calculando el índice de Zhang y Kanbur, tomando a San Salvador contra el resto de departamentos, los resultados nos muestran que existe una polarización entre el campo y la ciudad que no se ha modificado para el período en estudio, esto queda evidenciado en la gráfica 1.7 b. Esta gráfica reafirma, además, un matiz interesante; los grupos se mantienen, es decir, la relación campo-ciudad, pero además los niveles prácticamente no se han movido, lo que sí sucedía en el ingreso (gráfica 1.4 b), donde a pesar de que los grupos se mantenían, los desplazamientos a la derecha indican un incremento de los niveles de ingreso. De este modo los niveles de productividad no se incrementan de forma significativa en los grupos, lo cual encadena y limita más el movimiento de una posible distribución futura de ingreso regional.

\section{Matriz de transición 2. Productividad. El Salvador 1998-2011}

\begin{tabular}{|c|c|c|c|c|c|}
\hline & $\mathbf{0 - 7 2} \%$ & $\mathbf{7 2} \% \mathbf{- 7 9} \%$ & $\mathbf{7 9} \% \mathbf{- ~ 8 4} \%$ & $\mathbf{8 4} \% \mathbf{- 9 4} \%$ & $\mathbf{9 4} \%-\infty$ \\
\hline $\mathbf{3 8}$ & $\mathbf{0 . 6 8}$ & 0.16 & 0.11 & 0.03 & 0.03 \\
\hline $\mathbf{3 8}$ & 0.11 & $\mathbf{0 . 4 7}$ & 0.26 & 0.13 & 0.03 \\
\hline $\mathbf{3 6}$ & 0.08 & 0.25 & $\mathbf{0 . 3 3}$ & 0.33 & 0.00 \\
\hline $\mathbf{3 3}$ & 0.03 & 0.03 & 0.27 & $\mathbf{0 . 5 8}$ & 0.09 \\
\hline $\mathbf{3 7}$ & 0.03 & 0.05 & 0.00 & 0.03 & $\mathbf{0 . 8 9}$ \\
\hline $\mathbf{1 8 2}$ & & & & & \\
\hline Distribución Ergódica & $\mathbf{0 . 1 5}$ & $\mathbf{0 . 1 7}$ & $\mathbf{0 . 1 8}$ & $\mathbf{0 . 2 3}$ & $\mathbf{0 . 2 7}$ \\
\hline
\end{tabular}

Fuente: elaboración propia a partir de datos de EHPM (DIGESTYC, 1998-2011).

Como se ha mencionado, es necesario conocer para complementar el análisis de dicha variable la movilidad de los grupos que se dan dentro de la distribución. Y los resultados nos dicen que en el período de 1998-2011 ha existido menor movilidad esto según los índices de Shorrocks y Bartholomew, y se observa que al largo plazo existirá una divergencia hacia la media salvadoreña, es decir, que no habrá un proceso de homogenización. Lo que nos indica que a los grupos se les hará muy difícil mejorar su situación en términos de productividad. 
Al observar la matriz de transición 2 , se tiene que el $50 \%$ de los departamentos estará por debajo del $84 \%$ de la productividad media. El $23 \%$ se acerca al intervalo de $84 \%-94 \%$, pero aún por debajo de ella, mientras un $27 \%$ de los departamentos estaría alrededor y encima de esta media. Dado el peso de las personas ocupadas en San Salvador, esto significa que, prácticamente, este sería el único departamento por encima de la media. Nuevamente, si se compara esta matriz con la del ingreso, tenemos razones para afirmar que la productividad estará marcando límites a los esfuerzos redistributivos, por lo que se hace necesario movilizar, desagrupar y homogeneizar la productividad si se quiere homologar regionalmente los ingresos por persona.

Resumiendo otras variables analizadas en el estudio más amplio. Las variables de empleo, hogares en situación de pobreza extrema y relativa observamos iguales resultados pero en magnitudes distintas; ya que en términos de concentración, observamos que el índice de Theil posee una tendencia decreciente. Lo que nos lleva a decir que la desigualdad en las tres variables ha disminuido, es decir, que están tendiendo a la convergencia, siempre recordando que estamos estrictamente a la igualdad por departamentos y no dentro de ellos.

En términos de polarización, el empleo y los hogares en pobreza relativa presentan una tendencia constante, sin cambio, lo que nos lleva a decir que la polarización se ha mantenido en los mismos niveles. En cambio los hogares con pobreza extrema presentan una tendencia decreciente.

Al igual que el ingreso per cápita y productividad en estas variables, se realizó el análisis de concentración y polarización excluyendo al departamento de San Salvador y en las tres variables se llegó a la misma conclusión (en términos de tendencia). Al eliminar San Salvador, la desigualdad no cae, se mantiene. De este modo, la tendencia a disminuir o no la desigualdad pesa mucho si está o no San Salvador. Y en el análisis de polarización se determinó que San Salvador en empleo y en los hogares con pobreza extrema provoca un mayor grado de polarización. En los hogares con pobreza relativa al excluir a San Salvador el resto de los departamentos presentan una tendencia a crecer en desigualdad, es decir no solo se mantiene, sino que aumenta la desigualdad.

Y cuando observamos la tercera característica que es la movilidad. Observamos que la movilidad que obtuvimos en la matriz de transición según los índices Shorrocks y Bartholomew en la variable empleo, la movilidad en el periodo 19982011 presentó un ritmo desacelerado, mientras que en las variables de hogares con pobreza extrema y 
relativa la movilidad presenta una velocidad casi constante.

Según el vector ergódico en las tres variables, se espera que en el largo plazo exista una divergencia hacia la media salvadoreña se espera que no exista un proceso de homogenización, es decir, que a los individuos se les hará difícil mejorar su situación laboral y los hogares en pobreza extrema y relativa podrían tender a empeorar su situación.

Hay que destacar que la variable de hogares con remesas es la única que presenta resultados contrarios en dos de las tres características de análisis de desigualdad utilizadas en dicho trabajo.

En términos de concentración esta nos muestra una disminución en la desigualdad, es decir que dichos hogares están tendiendo a la convergencia. Pero cuando vemos el análisis de polarización, observamos que es la única variable que presenta un aumento en ella. Lo que nos lleva a decir que los grupos no se están igualando. Sin embargo, cuando se excluye en el análisis a San Salvador, de 1998 a 2006, pareciera que dicho departamento no tiene un impacto en los niveles de polarización. A partir del período comprendido entre 2006 y 2011, suceden dos situaciones. La primera, que San Salvador cobra mayor impacto en la polarización al incluirlo con el resto de departamentos. Y la segunda, que en el año 2011 el nivel de polarización es el mismo, ya sea excluyendo o no a dicho departamento. Al ver el análisis de movilidad, es la segunda característica donde se observa un resultado contrario a las demás. Primero la movilidad encontrada según los índices nos indica que esta ha tenido un ritmo constante, pero cuando observamos los resultados de los vectores ergódicos vemos que ésta en el largo plazo es la única variable que presenta una tendencia a la convergencia a la media salvadoreña lo que provocará una homogenización de los hogares con remesas, es decir que estos podrán mejorar su situación.

También se realizaron dos análisis más (aparte del análisis departamental), a nivel de ramas económicas en términos de concentración en un período de 1990 a 2011 y a nivel centroamericano en términos de concentración y polarización y para la productividad en un período de 1963 a 2010. Y las conclusiones a las que se llegaron son las siguientes: El PIB sectorial a precios constantes como a precios corrientes tiene una disminución de la desigualdad sectorial, al igual que a nivel departamental. Entonces el producto está repartiéndose más homogéneamente sectorialmente y departamentalmente, esto como grandes tendencias, pues a partir de 2007 se observa un incremento del nivel de desigualdad. Por otro lado, y en un total contraste, en Centroamérica con datos del EPWT (Extended Penn World Tables), v.4 se tiene una consistente desigualdad regional por país. 
La desigualdad y polarización parecen mejorar regionalmente. La desigualdad regional disminuye en muchas de las variables económicas analizadas y algo similar sucede con la polarización. Pero en la polarización o agrupamiento, dados los niveles altos de los que se parte, siguen persistiendo grupos y sobre todo la dicotomía campociudad. Asimismo, el departamento de San Salvador muestra una fuerte influencia en la existencia o no de la concentración y polarización. Por otro lado, debe advertirse que aunque parecen mejorar estos indicadores regionales, debe quedar claro que la falta de información municipal hace que el nivel de desigualdad y su tendencia pueda verse infravalorado y sesgado, pues lo que se mide es la desigualdad entre departamentos y se deja de medir la desigualdad al interior de ellos. De ahí la necesidad de observar desde varias perspectivas el fenómeno de la desigualdad.

Las matrices de transición (medidas en cinco intervalos) permiten indagar, medir y concluir que existe una movilidad regional pero que se desacelera, es decir, se mueven cada vez menos los departamentos que quieren revalorar su situación degradada, con la consecuencia de no mejorar, en esa medida, los niveles de desigualdad.

Pero, y más importante, el trabajo obtiene algunos rasgos escondidos de las distribuciones regionales. En una mirada a largo plazo, los vectores ergódicos nos muestran que no habrá convergencia para la mayoría de departamentos y por lo tanto, tampoco para sus habitantes. En materia de empleo, $78 \%$ de los departamentos estarán por debajo de las oportunidades medias de ocupaciones, en productividad el $73 \%$ de los departamentos y sus habitantes estarán por debajo de la media nacional, en pobreza relativa quedará encadenada poco más del $21 \%$ de la población y en pobreza extrema el $20 \%$, mientras que en términos de renta el $67 \%$ de los departamentos estará en el largo plazo, en ese limbo que marcaba Keynes, por debajo de la media nacional. Quedarse solamente en el análisis de desigualdad regional, en ese sentido, es un error por ser insuficiente en el diagnóstico regional.

Podemos resumir que EI Salvador, a nivel departamental, está tendiendo a la convergencia al igual que en sus ramas económicas. Esto nos llevaría a pensar que el país está pasando por un buen momento, pero el panorama cambia cuando observamos aspectos como polarización, conceptos que rescatan una información valiosa, lo cual nos deja la conclusión de que los grupos permanecen y si dirigimos la perspectiva más allá de los periodos gubernamentales, al plano del largo plazo, las 
conclusiones cambian diametralmente, no se prevé una homogeneización regional. Lo anterior plantea el reto de discutir los mecanismos que incrementen las inversiones regionales de infraestructura de largo plazo para incrementar la productividad en esa medida y aspirar a encontrar los conductos de gobierno para distribuir mejor el ingreso.

\section{Referencias bibliográficas}

os Atkinson A. (1987). On the Measurement of Poverty. Econometrical, 55(4), 749-764.

os BCR, (2012) Producto Interno Bruto por Rama de Actividad Económica a Precios Constantes de 1990 Millones de Dólares. El Salvador. Banco Central de Reserva de El Salvador. Disponible en: http://www.bcr.gob. sv/bcrsite/?cdr=30\&lang=es

os BCR, (2012) Producto Interno Bruto por Rama de Actividad Económica a Precios Corrientes de 1990 Millones de Dólares. El Salvador. Banco Central de Reserva de El Salvador. Disponible en: http://www.bcr.gob. sv/bcrsite/?cdr=30\&lang=es

os Barro, R. J. y Xavier Sala-i-Martin (1992). Convergence. Journal of Political Economy 100(2), 223-251.

os Carrillo-Huerta, M. y Vázquez, H. (2005) Desigualdad y polarización en la distribución del ingreso salarial en México. Problemas del desarrollo: revista latinoamericana de economía. 36(141), 109-130.

os Cockshott, P. y Cottrell, A. (1993). Hacia un nuevo socialismo. Inglaterra, Nottingham.

os Córdova, A. (2000). Convergencia y crecimiento en Centroamérica. Realidad No. 77, 515-544

os Cortés, F. y Rubalcava, R. (1984). Técnicas estadísticas de la desigualdad social. México: FLACSO.

os Dalton, H. (1920). The Measurement of the inequality of Incomes. The Economic Journal. 30(119), 348-361.

os De la Fuente, A y Freire, María Jesus. (2000). Estructura Sectorial y Convergencia Regional. Revista de Economía Aplicada8(23), 189-205.

os Dirección General de Estadísticas y Censos, DIGESTYC. (1999). Encuesta de Hogares de Propósitos Múltiples 1998. El Salvador: Ministerio de Economía, DIGESTYC.

os DIGESTYC. (2005). Censo económico 2005. El Salvador: Ministerio de Economía, DIGESTYC.

os DIGESTYC. (2000 al 2012). Encuesta de Hogares de Propósitos Múltiples 
1999. El Salvador: Ministerio de Economía, DIGESTYC.

os Duclos, J. Y., Esteban, J., Ray, D. (2004). Polarization: Concepts, measurement, estimation. Econometrica. 72(6).

os Esquivel, G. (1999). Convergencia Regional en México, 1940-1995. El Trimestre Económico. 66(264), 725-761.

os Esteban, J. M., Ray, D. (1994). On the measurement of polarization. Econometrica. 62(4).

os Ezcurra, R. (2001). Convergencia y cambio estructural en la Unión Europea. Departamento de Economía Universidad de Navarra.

os Goerlich F. (1998). Desigualdad, Diversidad y Convergencia (Algunos) Instrumentos de medida. Valencia.

os Gradín, C. y Del Río, C. (2001). Desigualdad, Polarización y Pobreza en la Distribución de la renta en Galicia. Instituto de Estudios Económicos de Galicia-Fundación P. Barrié de la Maza, n 11, A Coruña.

os Quah, D. (1993). Galton's Fallacy and Tests of the Convergence Hypothesis. Scandinavian Journal of Economics, Diciembre 1993.

os Sala-i-Martin, X. (2000). Apuntes de crecimiento económico. Elsa Vila Artadi (tr.). Barcelona: Antonio Bosch.

os Sánchez, C. (2002). Productividad y concentración (en las manufacturas mexicanas 1970-1993). (Tesis de licenciatura). Facultad de Economía, Universidad Nacional Autónoma de México. México.

cos Silber, J (1989). Factor Components, Population Subgroups and the Computation of the Gini Index of Inequality. The Review of Economics and Statistics. Feb, 1, p. 107-115.

os Vázquez, G. (2010). Convergencia real en Centroamérica: Evidencia empírica para el período 1990-2005. Instituto Complutense de Estudios Internacionales, ICEI. (07/10).

os Villaverde, J. (2007) Crecimiento y Convergencia Regional en España. (Algunas) Causas del Cambio. Papeles de economía española. (111), 240-254.

os Wolfson, M. C. (1994). When Inequalities Diverge, May 1994. The American Economic Review. 84(2), 353-358. 
Notas:

- 1 De modo que $R_{M}$ resulta ser una función que es creciente con los niveles de desigualdad (condición Pigou-Dalton) y aumenta con una tasa ¿ de crecimiento (condición de cambio relativo) entre los limites 1 y $n$. 